\title{
The optical/NIR afterglow of GRB 111209A: Complex yet not unprecedented $\star, \star \star$
}

\author{
D. A. Kann ${ }^{1,2,3,4}$, P. Schady ${ }^{2}$, E. F. Olivares ${ }^{5}$, S. Klose ${ }^{1}$, A. Rossi ${ }^{6,1}$, D. A. Perley ${ }^{7,8,9}$, B. Zhang ${ }^{10}$, T. Krühler ${ }^{2,8,11}$, \\ J. Greiner ${ }^{2,3}$, A. Nicuesa Guelbenzu ${ }^{1}$, J. Elliott ${ }^{2,12}$, F. Knust ${ }^{2}$, Z. Cano ${ }^{4}$, R. Filgas ${ }^{13}$, E. Pian ${ }^{5,14}$, P. Mazzali ${ }^{15,16}$, \\ J. P. U. Fynbo ${ }^{8}$, G. Leloudas ${ }^{17,8}$, P. M. J. Afonso ${ }^{18}$, C. Delvaux ${ }^{2}$, J. F. Graham ${ }^{2}$, A. Rau ${ }^{2}$, S. Schmidl ${ }^{1}$, \\ S. Schulze ${ }^{19,20,21}$, M. Tanga ${ }^{2}$, A. C. Updike ${ }^{22}$, and K. Varela ${ }^{2}$ \\ (Affiliations can be found after the references)
}

Received 1 June 2017 / Accepted 3 May 2018

\begin{abstract}
Context. Afterglows of gamma-ray bursts (GRBs) are simple in the most basic model, but can show many complex features. The ultra-long duration GRB 111209A, one of the longest GRBs ever detected, also has the best-monitored afterglow in this rare class of GRBs.

Aims. We want to address the question whether GRB 111209A was a special event beyond its extreme duration alone, and whether it is a classical GRB or another kind of high-energy transient. The afterglow may yield significant clues.

Methods. We present afterglow photometry obtained in seven bands with the GROND imager as well as in further seven bands with the Ultraviolet/Optical Telescope (UVOT) on-board the Neil Gehrels Swift Observatory. The light curve is analysed by multi-band modelling and joint fitting with power-laws and broken power-laws, and we use the contemporaneous GROND data to study the evolution of the spectral energy distribution. We compare the optical afterglow to a large ensemble we have analysed in earlier works, and especially to that of another ultra-long event, GRB 130925A. We furthermore undertake a photometric study of the host galaxy.

Results. We find a strong, chromatic rebrightening event at $\approx 0.8$ days after the GRB, during which the spectral slope becomes redder. After this, the light curve decays achromatically, with evidence for a break at about 9 days after the trigger. The afterglow luminosity is found to not be exceptional. We find that a double-jet model is able to explain the chromatic rebrightening. The afterglow features have been detected in other events and are not unique.

Conclusions. The duration aside, the GRB prompt emission and afterglow parameters of GRB 111209A are in agreement with the known distributions for these parameters. While the central engine of this event may differ from that of classical GRBs, there are multiple lines of evidence pointing to GRB 111209A resulting from the core-collapse of a massive star with a stripped envelope.
\end{abstract}

Key words. gamma-ray burst: general - gamma-ray burst: individual: GRB 111209A - gamma-ray burst: individual: GRB 130925A

\section{Introduction}

Gamma-ray bursts (GRBs) were discovered 50 years ago (Klebesadel et al. 1973) and represent the brightest explosions in the Universe, at cosmological redshifts (Metzger et al. 1997). One class, long GRBs, is associated with the core-collapse of very massive stars (see Woosley \& Bloom 2006; Hjorth \& Bloom 2012, and Cano et al. 2017, for reviews). These are accompanied by afterglow emission (van Paradijs et al. 1997; Costa et al. 1997; Frail et al. 1997) which can be extremely luminous (Akerlof et al. 1999; Kann et al. 2007; Racusin et al. 2008; Bloom et al. 2009; Woźniak et al. 2009; Perley et al. 2011; Vestrand et al. 2014). In the simplest models, the afterglow is pure synchrotron radiation (Sari et al. 1998; Rossi et al. 2011) and can be described by a smoothly broken power-law (e.g. Beuermann et al. 1999), the break to a steeper decay stemming from the collimation of the jet (Rhoads 1997, 1999; Sari et al. 1999).

Much focus in the last years has been on the divide (Mazets et al. 1981; Kouveliotou et al. 1993; Zhang et al. 2009)

* Partially based on observations obtained under programme 088.A0051(C), PI: J. P. U. Fynbo.

$\star \star$ The UVOT and GROND photometry (Table 2) is only available at the CDS via anonymous ftp to http://cdsarc.u-strasbg.fr (130.79.128.5) or via http://cdsarc.u-strasbg.fr/viz-bin/ qcat?] $/ \mathrm{A}+\mathrm{A} / 617 / \mathrm{A} 122$ between long and short GRBs (or, more generally, those not associated with the core-collapse of massive stars), but the other end of the duration distribution is also of great interest, as they pose challenges to the collapsar model of long GRBs (Woosley 1993; MacFadyen \& Woosley 1999). The duration of a GRB (once corrected for time dilation) generally reflects the duration of the central engine activity after subtracting the time it takes for the jet to break out of the star (e.g. Bromberg et al. 2012, 2013; Lazzati et al. 2013; Hamidani et al. 2017, and references therein). These latter publications find a typical engine activity time of $20 \mathrm{~s}$ which is far less than the duration of the longest known GRBs. The actual duration measurement is detector-dependent, though, and Zhang et al. (2014) pointed out that the true duration, which they label $t_{\text {burst }}$, is that of any detectable central engine activity (Liang et al. 2006), which may last much longer than the detectable gamma-rays (but see Boër et al. 2015a for a critical take on $t_{\text {burst }}$ ). Such an extreme duration was already discussed by Zou et al. (2006) in the context of the strong flaring of GRB 050904, which lasts $\gtrsim 7500 \mathrm{~s}$ in the rest-frame.

A second aspect which makes such extremely long GRBs interesting is that with modern rapid follow-up observations, both space- and ground-based, GRBs of long duration are still ongoing by the time they become observable by narrow-field instruments. Such observations have yielded the detection of optical flashes 
that are likely to be directly linked to the prompt emission itself (Blake et al. 2005; Vestrand et al. 2005, 2006, 2014; Page et al. 2007; Krühler et al. 2009; Beskin et al. 2010; Thöne et al. 2010; Guidorzi et al. 2011a; Greiner et al. 2014; Elliott et al. 2014).

Morphologically, the prompt emission light curves of GRBs are highly diverse, and this is especially true for extremely long GRBs. Different examples may be roughly sorted into five categories, which could indicate a link to different outflow structures from the central engine, or suggest that different processes are responsible for the dominating part of the prompt emission. We give examples of such events, as well as a (rough) phenomenological classification scheme in Appendix B. Some further possible extremely long GRBs have been discussed in Lien et al. (2016), based on BAT survey mode detections.

Levan et al. (2014, henceforth L14, see also Levan 2015) proposed that even among such events of extremely long duration, a few very rare cases exist which they label "ultra-long duration GRBs" (ULGRBs), and which may comprise a new class, possibly resulting from novel progenitor channels. Since then, this point has been debated. Zhang et al. (2014) found more cases of long-lasting central engine activity (though fainter and softer, and therefore not detected in gamma-rays) and no compelling evidence of a separate population in the $t_{\text {burst }}$-duration distribution (though conversely also no strong evidence against such a new population). A similar conclusion was reached by Virgili et al. (2013), but refuted by Boër et al. (2015a, see also Boër et al. 2015b) and Evans et al. (2014). Gao \& Mészáros (2015) also found a bimodal distribution and therefore the possibility that ULGRBs may be a different class, a result they further strengthened in a new paper (Gao et al. 2017). The most extreme of the three ${ }^{1}$ events that L14 described is GRB 111209A.

In terms of classical GRBs, even when taking the other events listed in Appendix B into account, GRB 111209A is an extreme case. Until recently, it was nothing less than the longest GRB detected so far (Gendre et al. 2013); a title now held by GRB 170714A (Appendix B). We refer to Appendix A for details on the early multi-wavelength follow-up of this event.

The initial behaviour in the optical and especially the X-rays (Appendix A) led to a comparison with both XRF 060218/SN 2006aj as well as the "Christmas Burst" GRB 101225A (Hoversten et al. 2011a) and it was also speculated that the event may be a further example of a tidal disruption flare like GRB 110328A/Swift J164449.3+573451 (Gendre et al. 2013; Levan et al. 2014). The extreme duration of the GRB and its apparent lack of a supernova have furthermore fuelled speculation that the progenitor may not have been a stripped-envelope star, but a very-low-metallicity blue supergiant (BSG) progenitor (Gendre et al. 2013; Levan et al. 2014; Stratta et al. 2013; Kashiyama et al. 2013; Nakauchi et al. 2013, see Kann et al. 2016, for more discussion).

In this paper, we present and discuss observations of the optical/near-infrared (NIR) afterglow of GRB 111209A obtained with GROND, the seven-channel Gamma-Ray burst Optical \& Near-infrared Detector (Greiner et al. 2008) mounted on the $2.2 \mathrm{~m}$ Max-Planck Gesellschaft/European Southern Observatory (MPG/ESO) telescope ${ }^{2}$ stationed in La Silla, Chile, as well as ultraviolet (UV)/optical data obtained with Ultraviolet/Optical Telescope (UVOT) on-board the Neil Gehrels Swift Observatory (see also L14, Stratta et al. 2013). While no observations during the prompt emission could be obtained with GROND due

\footnotetext{
1 Two further examples have been detected since then, GRBs 130925A and 170714A, see Appendix B.

2 The $2.2 \mathrm{~m} \mathrm{MPG}$ telescope starting on the 1st of October 2013.
}

to observing constraints, we discovered strong optical variability even during the following day, and followed the afterglow up regularly until it became unobservable, detecting the multiwavelength signature of late-time supernova emission.

Results on the supernova (SN) discovery, its spectroscopic verification, and modelling of the $\mathrm{SN}$, which has been designated SN 2011kl, are given in Greiner et al. (2015, henceforth G15). In this work, we present the entire GROND/UVOT data set, detailed afterglow and host-galaxy modelling, and place the entire event in the context of a large sample of GRB afterglows. In Kann et al. (2016, henceforth K18A), we also place SN $2011 \mathrm{kl}$ in the context of large SN samples, and combine all our results to discuss the nature of GRB 111209A.

The paper is organized as follows: in Sect. 2, we present the details of our observations and our data analysis. In Sect. 3, we present the results of fitting the light curve data, both temporally and as spectral energy distributions (SEDs), during the prompt emission and the afterglow phase; we also study the host galaxy and a very similar event, GRB 130925A. In Sect. 4, we model the strong chromatic rebrightening, study the energetics of the event and compare GRB 111209A to GRB 130925A, before finally concluding (Sect. 5). We present additional information on GRBs of extreme duration in the appendix.

We will follow the convention $F_{v} \propto t^{-\alpha} v^{-\beta}$ to describe the temporal and spectral evolution of the afterglow. We use WMAP $\Lambda$ CDM concordance cosmology (Spergel et al. 2003) with $H_{0}=71 \mathrm{~km} \mathrm{~s}^{-1} \mathrm{Mpc}^{-1}, \Omega_{\mathrm{M}}=0.27$, and $\Omega_{\Lambda}=0.73$. Uncertainties are given at $68 \%(1 \sigma)$ confidence level for one parameter of interest unless stated otherwise, whereas upper limits are given at the $3 \sigma$ confidence level.

\section{Observations}

\subsection{GROND observations}

At the time of the Swift trigger, GRB 111209A was just 13 degrees above the horizon as seen from La Silla and setting (Klotz et al. 2011a, 2011), which is under the pointing limit of the $2.2 \mathrm{~m}$ telescope, therefore no observations could be obtained during the extended prompt phase of the GRB with GROND. Our observations began the following night at the onset of astronomical twilight and continued for $5.5 \mathrm{~h}$, until the pointing limit of the telescope was reached. Analysis of the first observations yielded the detection of the optical/NIR afterglow at RA, $\operatorname{Dec}(\mathbf{J} 2000)=00: 57: 22.64,-46: 48: 03.6(14.34435,-46.80101)$ with an uncertainty of 0.3 (Kann et al. 2011a, see Fig. 1). Further analysis of the $J$-band observations of the first night also revealed the afterglow was actually rising in brightness and not falling (Kann \& Greiner 2011).

Observations continued during the following nights, in such a way as to create a dense follow-up on a logarithmic timescale. A total of 16 further epochs were obtained. Our penultimate observations were obtained on 18/19 February 2012, 72 days after the GRB; thereafter the position moved too close to the Sun and became unobservable. A final epoch was obtained after the GRB position had become visible again, 280 days after the GRB, to assess the host galaxy magnitude.

Afterglow magnitudes in the optical were measured against on-chip standard stars calibrated to the SDSS catalogue (Aihara et al. 2011), obtained from observing a nearby SDSS field immediately before the afterglow observations on the third day after the GRB, in photometric conditions. Nearinfrared magnitudes were measured against on-chip comparison stars taken from the 2MASS catalogue (Skrutskie et al. 2006). 


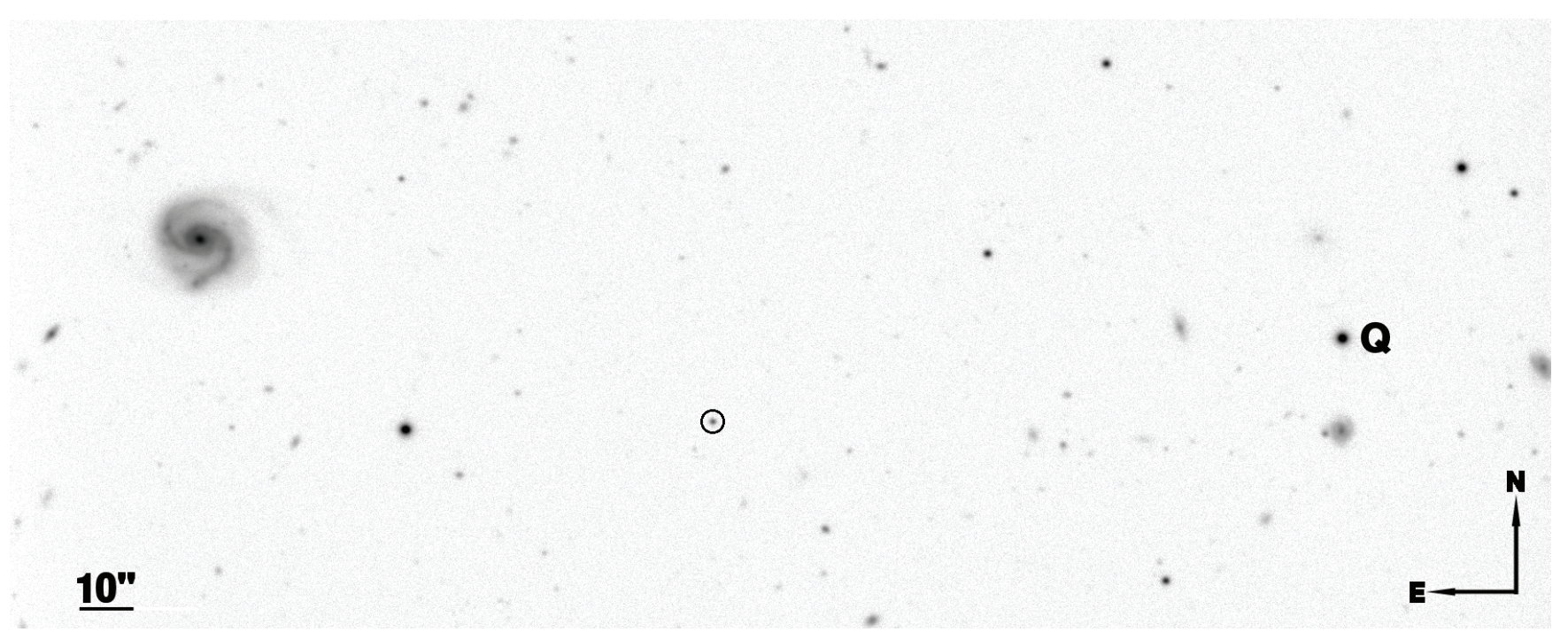

Fig. 1. Finding chart of the field of GRB 111209A; the afterglow is circled. This is a GROND $r^{\prime}$ image at 5.8 days post-burst; the afterglow has $r^{\prime}=21.2$. Seeing was $0^{\prime \prime} .73$ and the limiting magnitude is $r^{\prime}>25.3$. The field measures $4 .^{\prime} 9 \times 1.1^{\prime} 9$, pixel scale is $0^{\prime \prime} .158$ per pixel. We note the extreme sparsity of stars in the field; most visible sources are distant galaxies. The bright face-on spiral galaxy to the north-east is 6dFGS gJ005732.0-464730 at $z=0.08851$ (Jones et al. 2009). The character Q marks quasar [VV96] J005711.3-464750 at $z=2.0$ (Maza et al. 1993).

Reduction and analysis was performed within a custom pipeline calling upon IRAF tasks (Tody 1993), following the methods described in detail in Krühler et al. (2008) and Yoldaş et al. 2008.

\subsection{UVOT observations}

We expand our photometric database by adding the UVOT observations from Swift. UVOT photometry was carried out on pipeline-processed sky images downloaded from the Swift data centre $^{3}$ following the standard UVOT procedure (Poole et al. 2008). Source photometric measurements were extracted from the UVOT early-time event data and later imaging data files using the tool UvotmaGHIST (v1.1) with a circular source extraction region of 3".5 to maximise the signal-to-noise ratio $(\mathrm{S} / \mathrm{N})$. In order to remain compatible with the effective area calibrations, which are based on $5^{\prime \prime}$ aperture photometry (Poole et al. 2008), an aperture correction was applied. We note independent reductions of the UVOT data set have been used by Stratta et al. (2013) and L14. Our UVOT data results agree well with those of L14 where they overlap.

\subsection{X-shooter observations}

Two X-shooter (Vernet et al. 2011) spectra are included in L14. We downloaded and independently reduced these spectra; for details see Krühler et al. (2015). The afterglow spectrum, taken 0.74 days after the GRB, yields a precise redshift of $z=0.67702 \pm 0.00005$. The late spectrum, taken during the rising phase of the $\mathrm{SN}$ at 19.82 days after the GRB, is discussed in detail in G15.

\section{Results}

The GROND and UVOT data of the afterglow of GRB 111209A are given in table form at CDS (some of the late data have already been published in G15) and plotted in their entirety

\footnotetext{
3 www.swift.ac.uk/swift_portal
}

(with additional data from the literature) in Fig. 2. In the $r^{\prime} i^{\prime} z^{\prime}$ bands, the afterglow is detected in all 46 epochs, in the $g^{\prime}$ band, it is undetected in the last epoch before the host observation. In the NIR, the afterglow is detected in $J H$ until $2.4 \mathrm{Ms} / 27.79$ days (not in the last five epochs), and in the $K_{S}$ band, it is detected until 1.9 Ms/21.77 days (not in the last six epochs). Multi-colour observations and detections by UVOT extend to $\$ 500 \mathrm{ks} / 6$ days, and the afterglow/SN is detected until 2.2 Ms/25.28 days in white light. The data in the table are given in $\mathrm{AB}$ magnitudes and have not been corrected for Galactic foreground extinction. For the GRB position, the maps of Schlegel et al. (1998) yield $E_{(B-V)}=0.020 \mathrm{mag}$, using the Milky Way extinction curve of Cardelli et al. (1989) and the extinction coefficients for the GROND filters ${ }^{4}$, we derive small extinction corrections as given in the footnote of the data table at CDS, in other words, the influence of foreground extinction is almost negligible.

The afterglow light curve (Fig. 2) reveals multiple distinct phases in its evolution. First and foremost, there is strong variability seen in the UVOT data that is contemporaneous with the long-lasting prompt emission, before the afterglow transits to a constant decay phase (see also Stratta et al. 2013). Subsequently, during the GROND observations spanning the first night, the afterglow turns from a plateau (more specifically a shallow rise) phase to a very steep rise (Kann \& Greiner 2011; Gendre et al. 2013; Stratta et al. 2013). A day later, the GROND magnitudes of the afterglow are similar to those at the end of the GROND observations during the first night; they are then seen to decay steadily, implying a turnover must have occurred in between, which the UVOT data confirms. With the exception of two small "steps", the afterglow decays smoothly and without exhibiting any visible breaks, until at least 13 days. The afterglow departs from this decay in observations starting three weeks after the trigger, revealing a chromatic bump until the end of our observations. In the white data as well as the $F 336 \mathrm{~W} / u^{\prime}$ data from L14, the afterglow is seen to decay more rapidly.

4 It is $A_{g^{\prime}} / A_{\mathrm{V}}=1.255, A_{r^{\prime}} / A_{\mathrm{V}}=0.866, A_{i^{\prime}} / A_{\mathrm{V}}=0.648, A_{z^{\prime}} / A_{\mathrm{V}}=0.482$, $A_{J} / A_{\mathrm{V}}=0.283, A_{H} / A_{\mathrm{V}}=0.181, A_{K_{S}} / A_{\mathrm{V}}=0.116$. 


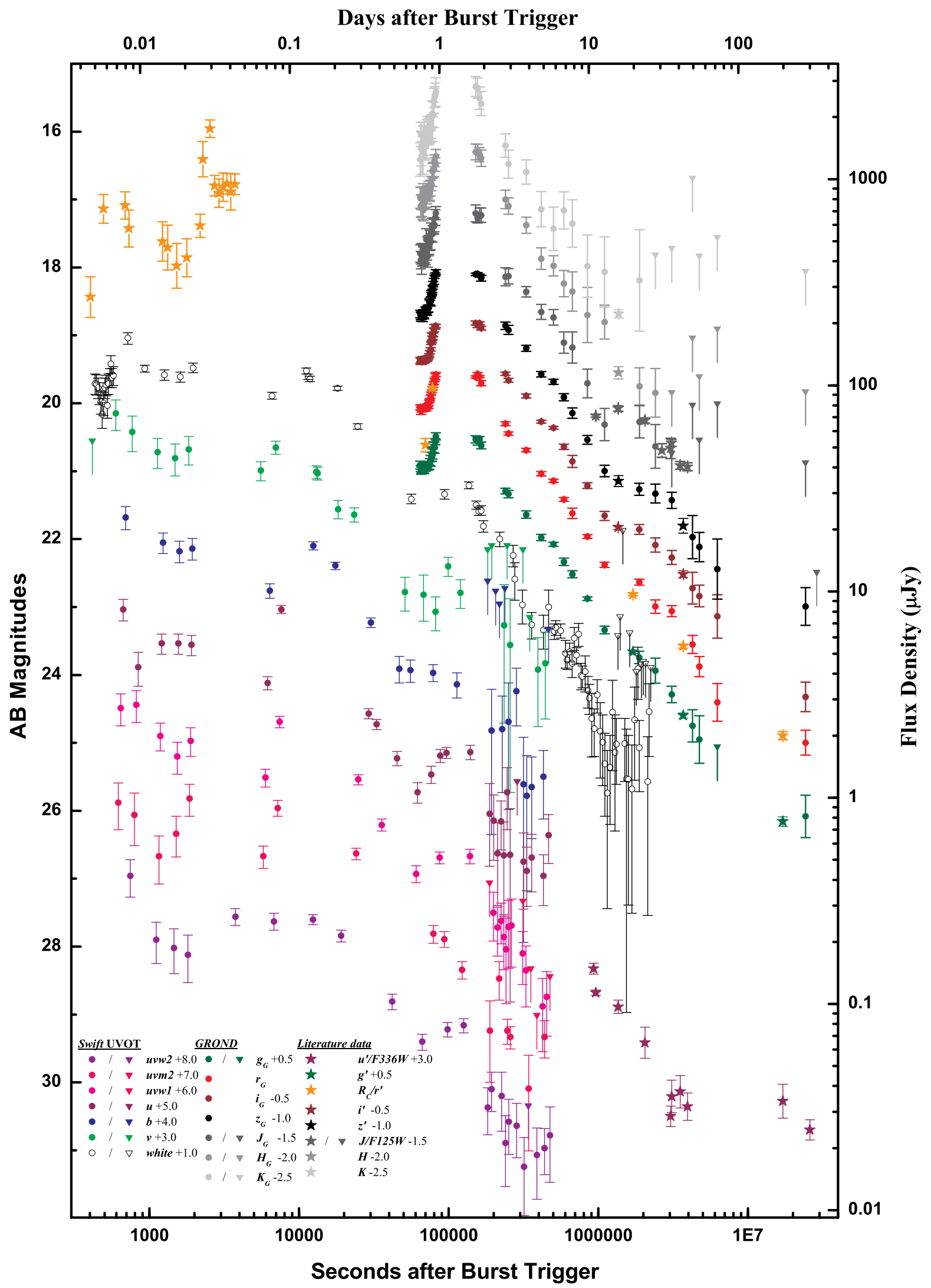

Fig. 2. Light curves of the optical counterpart of GRB 111209 A as observed by GROND and UVOT (this work), with additional $R_{C}$ data from Nysewander et al. (2011) and Stratta et al. (2013), and $F 336 W u^{\prime} g^{\prime} r^{\prime} R_{C} i^{\prime} z^{\prime} F 125 W J H K$ data from L14. Downward-pointing triangles are upper limits. The different bands are colour-coded and given in the legend; they are offset for clarity by the amount given. The $G$ subscript denotes GROND data. Data in this plot are corrected for the (small) Galactic foreground extinction. 


\subsection{Detailed light-curve fitting of the afterglow}

\subsubsection{The prompt emission}

The optical and X-ray emission (which shows evidence for a minor contribution from a blackbody, Gendre et al. 2013, L14) during the prompt phase has been discussed extensively by Stratta et al. (2013). We therefore simply discuss a few of the results. From 0.008 to 0.025 days (the first Swift orbit as well as some TAROT data from Stratta et al. 2013), all data are fit by an achromatic broken power-law:

$m(t)=-2.5 \log \left(10^{-0.4 m_{k}}\left(\left(\frac{t}{t_{\mathrm{b}}}\right)^{\alpha_{1} n}+\left(\frac{t}{t_{\mathrm{b}}}\right)^{\alpha_{2} n}\right)^{-1 / n}\right)$.

Here, $m_{k}$ is the normalization magnitude, $t$ the time after trigger, $\alpha_{1,2}$ the power-law slopes of the first and second component, $t_{\mathrm{b}}$ the break time, and $n$ the smoothness of the break. We ignore any host galaxy contribution since it lies far below the magnitudes we are dealing with here (Sect. 3.3). We find $\alpha_{1}=1.40 \pm 0.29, \alpha_{2}=-0.21 \pm 0.16, t_{\mathrm{b}}=1020 \pm 89 \mathrm{~s}$, $n=-10$ fixed $\left(\chi^{2}=20.1\right.$ for 31 degrees of freedom). This emission is relatively red, with a spectral slope $\beta=1.43 \pm 0.12$, in perfect agreement with Stratta et al. (2013), who find $\beta=$ $1.43 \pm 0.20$.

Our SED exhibits slight curvature, and the addition of NIR data from REM (Fugazza et al. 2011) allows an acceptable fit $\left(\chi^{2}=5.84\right.$ for 5 degrees of freedom) with a SMC dust model yielding an intrinsic spectral slope $\beta_{0}=0.63 \pm 0.24$ and restframe extinction $A_{\mathrm{V}}=0.25 \pm 0.11 \mathrm{mag}$. Stratta et al. (2013) note that the SED is redder than at later times (and also redder than typical pure GRB afterglows) and invoke possible dust destruction. We caution that while we find higher extinction at early times, the increase is only at the $1.2 \sigma$ level (see Sect. 3.2 ), and it is also not clear if the emission truly is an underlying powerlaw. Furthermore, as the GRB had already been on-going for several kiloseconds (Golenetskii et al. 2011), it would be peculiar to still detect further dust destruction and de-reddening; this process is expected to happen on much shorter timescales (Waxman \& Draine 2000; Starling 2008; Morgan et al. 2014). We also note that the temporal slopes we derive are arbitrary values dependent on the trigger time of Swift. If $t_{0}$ is set significantly earlier, to the beginning of the Konus-WIND detection, the slopes become significantly steeper.

The afterglow is further observed by TAROT (Stratta et al. 2013), who find a strong flare (see Lin et al. 2018 for an interpretation of the X-ray-to-optical delay of this flare). The UVOT data from the second Swift orbit can be described by the transition from a decay to another steep flare assuming the colours are identical to those we find from the first orbit; a similar result is also derived by Stratta et al. (2013). The following orbit may also show a decay followed by a rise; Stratta et al. (2013) interpret this behaviour as the onset of the forward-shock afterglow. At this time, the X-ray emission begins a steeper decay, but it does not yet transition to the very steep decay dominated by high-latitude emission which marks the end of the prompt emission.

\subsubsection{The early afterglow}

The UVOT data from 0.15 to 0.75 days can be fit by an achromatic power-law decay. We find $\alpha=1.23 \pm 0.05 \quad\left(\chi^{2}=13.57\right.$ for 16 degrees of freedom), and a six-colour SED with a small amount of scatter and no evidence for curvature, adequately $\left(\chi^{2}=4.64\right.$ for 4 degrees of freedom) fit by a simple power-law with slope $\beta=0.96 \pm 0.14$. We note that these values differ relatively strongly from those presented in Stratta et al. (2013), who find $\alpha=1.6 \pm 0.1, \beta=1.33 \pm 0.01$. Our results indicate a transition to a bluer spectrum compared to the first orbits, and the smooth decay is indicative of this being a forward-shock afterglow. During this phase, the X-ray afterglow behaves differently, transitioning to a steep decay which is seen both by Swift $/ \mathrm{XRT}^{5}\left(\alpha_{\mathrm{X} 1, \mathrm{XRT}}=6.45_{-0.15}^{+0.13}\right)$ and XMM-Newton $\left(\alpha_{\mathrm{X} 1, \mathrm{XMM}}=2.23 \pm 0.10\right.$, Stratta et al. 2013). This differing behaviour may indicate that the optical emission associated with the prompt GRB emission is significantly fainter than the afterglow, whereas the tail of the prompt emission still dominates the X-ray regime.

\subsubsection{The first day}

GROND observations begin 0.75 days after the trigger, and we find that the first night of data is described by a (slightly rising) plateau phase followed by a steep rise, first reported by Kann \& Greiner (2011). In this case, the values of $\alpha_{1,2}$ will be negative. Furthermore, since the broken power-law is a convex function in this case, the smoothness $n$ must be negative as well; we are not able to let it be a free parameter see, (e.g. Zeh et al. 2006, on $n$ as a free parameter in light curve fits or not), and we fix it to a hard value, $n=-1000$, as we find no evidence for a smoother rollover and a hard $n$ minimizes the errors of the temporal slopes. Usually, $n=(-) 10$ is already considered a hard transition (Zeh et al. 2006), but in this case, we are working with very short baselines in $\log t$ space, that is, the data points do not extend far enough before and after the break. Using $n=-10$ would result in power-law indices that are not yet very close to their asymptotic values, leading to much larger variations and errors. This is mitigated by using $n=-1000$, an abrupt transition between the two slopes. The addition of UVOT data during this phase does not lead to any improvement of the results, as the data is sparse and suffers from scatter.

We performed two different fits. In the first case, we performed a joint fit with all seven bands, leaving the slopes and the break time free to vary, but using only one shared value between all seven bands. This fit yields a satisfactory result, as given in Table 3. Motivated by our SED results (Sect. 3.2), we also performed broken power-law fits for each band separately; these results are also given in Table 3, and the fit is shown in Fig. 3. The fit finds different values for each of the bands. A detailed look at the differences between filters reveals a span between essentially no differences and $2 \sigma$ offsets. This weak evidence for chromatic evolution is in agreement with the good fit derived using shared parameters. The initial evolution is close to flat in $g^{\prime} r^{\prime} i^{\prime}$, but becomes steeper for redder bands, and this is also true for the very steep rise starting at $\approx 0.86$ days. Indeed, the slope difference $\Delta \alpha$ remains constant within errors over all bands, though we highlight that the errors, especially in the $K_{S}$ band, are very large. The break time $t_{\mathrm{b}}$ is constant in all bands within $2 \sigma$. While the evidence for this chromatic change is not strong from these fits,

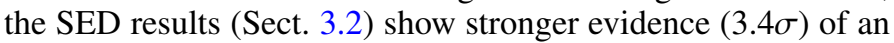
intrinsic slope change over this rebrightening phase, indicating that the per-band fit is physically preferred.

\subsubsection{The transition from steep rise to standard decay}

The following night, the afterglow (as measured by GROND) was at a similar magnitude as at the end of the first night's observations, but had started to decay, which became clear over

http://www.swift.ac.uk/xrt_live_cat/00509336/ 
Table 1. Local standard stars.

\begin{tabular}{ccccccccc}
\hline \hline RA $(\mathrm{J} 2000)$ & Dec $(\mathrm{J} 2000)$ & $g^{\prime}(\mathrm{AB} \mathrm{mag})$ & $r^{\prime}(\mathrm{AB} \mathrm{mag})$ & $i^{\prime}(\mathrm{AB} \mathrm{mag})$ & $z^{\prime}(\mathrm{AB} \mathrm{mag})$ & $J(\mathrm{Vega} \mathrm{mag})$ & $H(\mathrm{Vega} \mathrm{mag})$ & $K(\mathrm{Vega} \mathrm{mag})$ \\
\hline $00: 57: 09.09$ & $-46: 47: 16.4$ & $19.985 \pm 0.008$ & $18.440 \pm 0.009$ & $16.954 \pm 0.007$ & $16.266 \pm 0.006$ & $14.743 \pm 0.015$ & $14.183 \pm 0.021$ & $13.981 \pm 0.031$ \\
00:57:14.44 & $-46: 48: 33.1$ & $21.452 \pm 0.012$ & $20.039 \pm 0.011$ & $19.103 \pm 0.009$ & $18.734 \pm 0.009$ & $17.448 \pm 0.027$ & $16.760 \pm 0.034$ & $16.705 \pm 0.074$ \\
00:57:15.53 & $-46: 46: 57.0$ & $21.191 \pm 0.012$ & $19.803 \pm 0.010$ & $18.475 \pm 0.008$ & $17.922 \pm 0.007$ & $16.490 \pm 0.020$ & $15.902 \pm 0.026$ & $15.653 \pm 0.040$ \\
$00: 57: 17.67$ & $-46: 47: 32.4$ & $21.550 \pm 0.014$ & $20.027 \pm 0.010$ & $18.479 \pm 0.008$ & $17.830 \pm 0.006$ & $16.389 \pm 0.018$ & $15.861 \pm 0.024$ & $15.566 \pm 0.040$ \\
00:57:28.21 & $-46: 48: 05.2$ & $18.390 \pm 0.004$ & $17.736 \pm 0.009$ & $17.483 \pm 0.009$ & $17.400 \pm 0.007$ & $16.389 \pm 0.018$ & $15.830 \pm 0.024$ & $15.678 \pm 0.044$ \\
00:57:30.35 & $-46: 51: 09.6$ & $21.661 \pm 0.016$ & $20.246 \pm 0.012$ & $19.377 \pm 0.012$ & $19.086 \pm 0.011$ & $17.749 \pm 0.031$ & $17.184 \pm 0.047$ & $16.818 \pm 0.094$ \\
00:57:32.34 & $-46: 51: 24.8$ & $20.147 \pm 0.007$ & $19.418 \pm 0.010$ & $19.096 \pm 0.012$ & $19.016 \pm 0.012$ & $17.955 \pm 0.035$ & $17.415 \pm 0.047$ & $17.435 \pm 0.143$ \\
\hline
\end{tabular}

Notes. Magnitudes are given in the native filter system.

Table 3. Slopes of the plateau/rebrightening phase of the optical/NIR afterglow of GRB 111209A (see Fig. 3).

\begin{tabular}{lcclc}
\hline \hline Filter & $\alpha_{1}$ & $\alpha_{2}$ & $\begin{array}{l}\text { Break } \\
\text { time }(\mathrm{ks})\end{array}$ & $\chi^{2}$ \\
\hline$g^{\prime}$ & $-0.29 \pm 0.28$ & $-3.65 \pm 0.56$ & $74.0 \pm 1.0$ & 4.16 \\
$r^{\prime}$ & $-0.26 \pm 0.19$ & $-3.73 \pm 0.20$ & $73.0 \pm 0.4$ & 5.96 \\
$i^{\prime}$ & $-0.17 \pm 0.20$ & $-4.16 \pm 0.19$ & $72.7 \pm 0.3$ & 12.05 \\
$z^{\prime}$ & $-0.78 \pm 0.35$ & $-4.43 \pm 0.30$ & $73.0 \pm 0.7$ & 6.26 \\
$J$ & $-1.07 \pm 0.89$ & $-4.06 \pm 0.94$ & $73.5 \pm 2.5$ & 4.39 \\
$H$ & $-1.22 \pm 0.69$ & $-5.02 \pm 1.21$ & $74.7 \pm 1.9$ & 3.89 \\
$K_{S}$ & $-1.78 \pm 0.77$ & $-7.59 \pm 3.28$ & $76.7 \pm 2.1$ & 5.80 \\
All & $-0.34 \pm 0.12$ & $-4.07 \pm 0.12$ & $72.98 \pm 0.23$ & 94.54 \\
\hline
\end{tabular}

Notes. The data spans from $64 \mathrm{ks}$ to $82 \mathrm{ks}$. The degrees of freedom for each single-filter fit are 23. For the joint fit, the degrees of freedom are 179 .

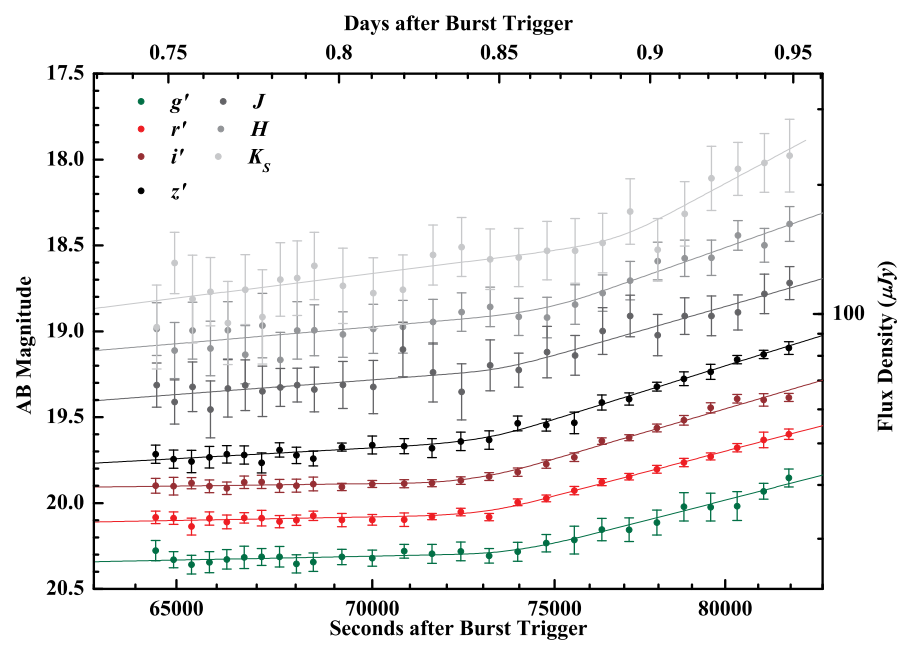

Fig. 3. Fit to the light curves of the afterglow of GRB 111209A during the first night of observations. The slopes and break times are left free to vary in each band. See Table 3 for values.

the following nights. Therefore, a transition must have occurred in between, for which we have no GROND data (but UVOT data, see below). The simplest solution is that the afterglow rises to a peak and rolls over into the later decay phase, implying only a single break. Such behaviour is well-known from early afterglow observations as the "rising of the forward shock" (e.g. Vestrand et al. 2006; Molinari et al. 2007; Krühler et al. 2008; Ferrero et al. 2009; Oates et al. 2009), but is rarely seen at such late times after the trigger. We find that the decay following the

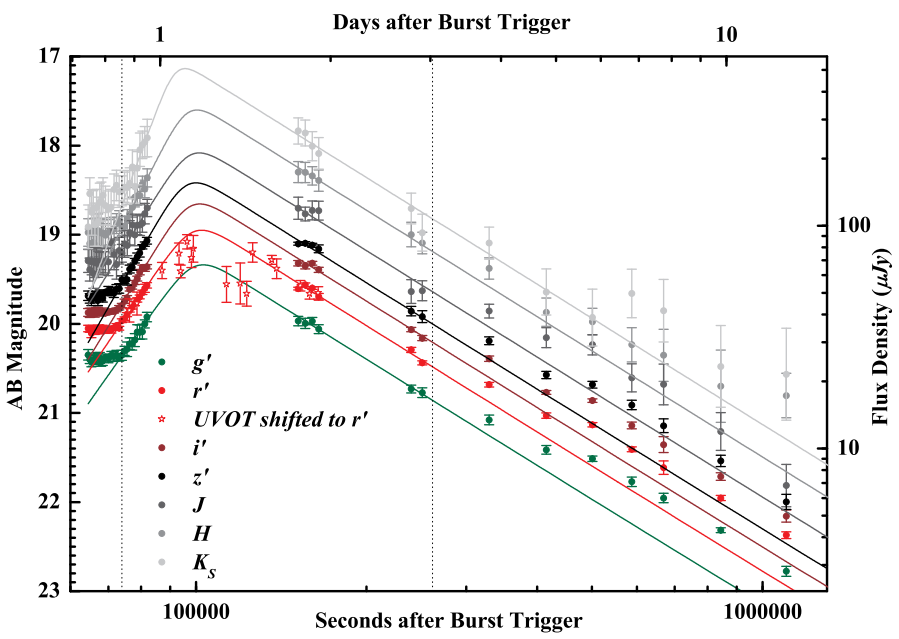

Fig. 4. Fit to the light curves of the afterglow of GRB 111209A from the beginning of the rebrightening at $\approx 74$ ks to before the "step" at about three days (between the vertical dotted lines). This fit uses GROND data only and is the simplest possible transition between the rising and decaying regimes. The stars represent UVOT data (multiple colours) shifted to the $r^{\prime}$ band. This shows that the evolution over the peak is possibly more complicated than our simple model. The decay slope after the peak is a free but shared parameter for all seven bands, whereas the index of the rise is different for each band. See Table 4 for values.

possible peak is achromatic (though there is some scatter), and therefore we declare $\alpha_{3}$ to be a shared but otherwise free parameter for all bands. The slope of the steep rise as well as the break time are left free and individual for all bands. We fix the smoothness to $n=5$, as such transitions are usually not sharp, but note that since we only fit data which lie relatively far from the peak, the actual result is independent of the chosen smoothness. Following Molinari et al. (2007), the break time is only identical to the peak time for a symmetric rise and decay, which we clearly do not see here. Therefore the actual peak times are determined by (in our case, we label the rise $\alpha_{2}$ and the decay $\alpha_{3}$ ):

$t_{\text {peak }}=t_{\mathrm{b}}\left(\left(-\alpha_{2} / \alpha_{3}\right)^{1 /\left[n\left(\alpha_{3}-\alpha_{2}\right)\right]}\right)$.

The fit is shown in Fig. 4 and the results are given in Table 4. The $\alpha_{2}$ values that we find from this fit are in excellent agreement with those given in Table 3. The peak times are identical within errors; the only slight outlier is the $K_{S}$ band, but here again the errors are larger. We only fit the data until $\approx 3$ days after the GRB, as afterwards, there is a clear "step"; the first of two (see below).

This phase between the two GROND epochs is, however, covered by UVOT. Again, the data show large errors and scatter, but shifting the higher-S/N points to the $r^{\prime}$ band reveals that our 
Table 4. Slopes of the rebrightening and first decay phase of the optical/NIR afterglow of GRB 111209A, as well as the peak time and magnitudes.

\begin{tabular}{lrrc}
\hline \hline Filter & \multicolumn{1}{c}{$\alpha_{2}$} & $t_{\text {peak }}(\mathrm{ks})$ & Peak magnitude $(\mathrm{AB})$ \\
\hline$g^{\prime}$ & $-3.62 \pm 0.48$ & $102.7_{-3.2}^{+3.4}$ & $19.33 \pm 0.04$ \\
$r^{\prime}$ & $-3.74 \pm 0.18$ & $102.1_{-1.3}^{+1.4}$ & $18.94 \pm 0.02$ \\
$i^{\prime}$ & $-4.17 \pm 0.19$ & $101.2 \pm 1.2$ & $18.64 \pm 0.02$ \\
$z^{\prime}$ & $-4.45 \pm 0.28$ & $99.6_{-1.4}^{+1.5}$ & $18.42 \pm 0.02$ \\
$J$ & $-4.08 \pm 0.79$ & $101.1_{-4.5}^{+4.8}$ & $18.08 \pm 0.07$ \\
$H$ & $-5.01 \pm 0.90$ & $100.1_{-4.0}^{+4.3}$ & $17.59 \pm 0.06$ \\
$K_{S}$ & $-6.98 \pm 2.16$ & $95.2_{-5.1}^{+5.9}$ & $17.13 \pm 0.09$ \\
\hline
\end{tabular}

Notes. It is $\alpha_{3}=1.59 \pm 0.03$ for all bands, and $\chi^{2}=70$ for 90 degrees of freedom.

simple model may not represent reality. A cluster of data points lies beneath our model fit (see Fig. 4), indicating that there may be an earlier turnover followed by another strong rebrightening that has ended by the time GROND starts observing during the second night. The sparsity of the data, however, does not allow further conclusions.

This period is mostly covered by high-S/N XMM-Newton X-ray observations, as has been mentioned (Gendre et al. 2013; Stratta et al. 2013). These data show no rebrightening as seen in the optical. Instead, after the end of the steep decay (Sect. 3.1.2), the X-rays show a plateau $\left(\alpha_{\mathrm{X} 2, \mathrm{XMM}}=0.18 \pm 0.05\right)$, which breaks into a regular decay $\left(\alpha_{\mathrm{X} 3, \mathrm{XMM}}=1.52 \pm 0.06\right.$, we note this value is in excellent agreement with our late-time slope in the optical/NIR). A blackbody component as in the early prompt emission is no longer detected (Gendre et al. 2013), but Stratta et al. (2013) report an additional hard spectral component.

Figure 4 shows that at $\approx 4$ days, the magnitude of the afterglow increases, though we do not observe the actual transition. Fitting only epochs $34 / 35$ (at $\approx 4-5$ days) yields $\alpha_{3.2}=1.44 \pm 0.09$ and $\chi^{2}=1.5$ for 8 degrees of freedom. Thereafter, the afterglow "steps up" yet again. In the $r^{\prime}$ band, each of the steps represents a brightening by $\approx 0.2 \mathrm{mag}$. We note that our slope values are in agreement with those found by Stratta et al. $(2013, \approx 1.5-1.6)$.

\subsubsection{The late afterglow (and the supernova)}

Data beyond six days show no more deviation from a smooth decay (i.e. another "step" rebrightening) until the supernova becomes dominant in the optical/NIR bands. The fit to the entire optical/NIR afterglow (after the rebrightening; taking the "steps" into account), the supernova component, and the host galaxy (Sect. 3.3) was first given in G15, and is discussed in detail in K18A. To summarise, we find from the ultraviolet data, to which the supernova contributes negligibly, that the afterglow in this segment reveals a light-curve break, and we find $\alpha_{\text {late }, 1}=1.55 \pm 0.01, \alpha_{\text {late }, 2}=2.33 \pm 0.16, t_{\mathrm{b}}=9.12 \pm 0.47$ days; $n=10$ has been fixed. We have also performed a $u^{\prime}$-band-only fit, and recover the broken power-law fit, $\alpha_{\text {late }, u, 1}=1.61 \pm 0.18$, $\alpha_{\text {late }, u, 2}=2.30 \pm 0.27, t_{\mathrm{b}}=6.45 \pm 2.71$ days $(n=10$ fixed $)$. The earlier break time is likely caused by lack of data from 5.4 to 10.8 days, but in the joint fit, the unbroken $g^{\prime} r^{\prime} i^{\prime} z^{\prime}$ data imply a later break. An attempt to find this break also in the UVOT white-band data does not yield conclusive results. While there seems to be a steepening (Fig. 2), the data at $>10$ days are affected by strong scatter. We note that as the white bandpass encompasses the entire CCD response of UVOT, it must also contain SN light, as it extends to the $g^{\prime} V$ filters, where the $\mathrm{SN}$ is clearly detected. Therefore, the late white light curve has an unknown SN contribution which we cannot take into account, but which will somewhat flatten the light curve again.

The steepening of the decay, as shown in Fig. 1 of K18A and $\mathrm{G} 15$, is not easy to decipher visually. Two main reasons for this are the following. For one, the magnitude difference between the afterglow at break time and the host galaxy is not particularly large, $\approx 2$ mag, implying the host contributes strongly to the post-break brightness of the transient (i.e. afterglow plus host). G15 show the light-curve decomposition in the $u$ band, where the steep decay becomes evident. Secondly, the slope change $\Delta \alpha=0.78 \pm 0.16$ is relatively shallow, as a comparison with the sample presented in Zeh et al. (2006, their Fig. 3) shows (barely in the second-lowest bin). GRB afterglows with comparable decay slopes presented in that work are GRBs 000926 and 020124 (\#6 and \#10 in Fig. 1 of Zeh et al. 2006, respectively); in both cases, the slope change is subtle and not easily visible (both GRB afterglows show a larger $m_{\text {break }}$ to $m_{\text {host }}$ contrast, though).

\subsection{The evolution of the spectral slope}

Multiple photometric measurements of a GRB afterglow obtained at a similar point in time (or corrected for the evolution of the afterglow) yield a spectral energy distribution (SED), a verylow-resolution spectrum. The spectrum of a GRB afterglow is intrinsically a power-law due to the synchrotron-radiation nature of the emission (Sari et al. 1998), but a curvature can be induced by line-of-sight dust in the host galaxy even after the correction for Galactic extinction (e.g. Fynbo et al. 2001; Kann et al. 2006, 2010). The afterglow evolution is generally achromatic, a result that led Kann et al. (2010) to create compound light curves by shifting data of other filters to the $R_{C}$ band. Significant evidence for colour evolution of afterglows was quite sparse until recently; some well-known examples show that it is typically found at early times, and only if a bright afterglow leads to dense multi-wavelength sampling, which is the case for such examples as GRB 030329 (Lipkin et al. 2004), GRB 061126 (Perley et al. 2008a; Gomboc et al. 2008) GRB 080319B (Bloom et al. 2009; Racusin et al. 2008; Woźniak et al. 2009) and GRB 130427A (Panaitescu et al. 2013; Laskar et al. 2013; Vestrand et al. 2014; Maselli et al. 2014; Perley et al. 2014).

GROND, due to its inherent simultaneous seven-colour observation mode, is predestined to study the SEDs of GRB afterglows, as each observation yields a seven-band SED with no concerns about variability. Combined with the ability to respond to GRBs within minutes, and the general collecting power of a $2.2 \mathrm{~m}$ telescope, it is no wonder that GROND has allowed the detailed study of multiple SEDs for several GRB afterglows and has allowed the discovery of significant spectral evolution in many cases; some examples are XRF 071031 (Krühler et al. 2009), GRB 080129 (Greiner et al. 2009), GRB 080413B (Filgas et al. 2011b), GRB 081029 (Nardini et al. 2011), GRB 100814A (Nardini et al. 2014), GRB 100621A (Greiner et al. 2013) and GRB 091127 (Filgas et al. 2011a), the latter even exhibiting smooth spectral evolution without any actual afterglow variability.

From the UVOT (as well as TAROT and REM during the prompt emission) data, we have already derived two SEDs (Sects. 3.1.1 and 3.1.2), both of which needed joint multi-colour fits over moderately long temporal baselines to yield viable results. For GROND, each "shot" gives us one SED. 
Table 5. Spectral slope $\beta$ of the optical/NIR afterglow of GRB 111209A over time.

\begin{tabular}{|c|c|c|c|c|}
\hline$\Delta \mathrm{t}(\mathrm{s})$ & $\beta$ & $A_{\mathrm{V}}$ & Data set & $\chi_{v}^{2}$ \\
\hline $572-2176$ & $1.176 \pm 0.078$ & $\ldots$ & UVOT+TAROT+REM & 2.24 \\
\hline $572-2176$ & $0.634 \pm 0.250$ & $0.253 \pm 0.105$ & UVOT+TAROT+REM & 1.17 \\
\hline $12428-67823$ & $0.956 \pm 0.140$ & $\ldots$ & UVOT & 1.16 \\
\hline 64492 & $0.924 \pm 0.074$ & $\cdots$ & GROND & 0.26 \\
\hline 64929 & $0.962 \pm 0.066$ & $\ldots$ & GROND & 0.41 \\
\hline 65373 & $1.015 \pm 0.076$ & $\cdots$ & GROND & 0.13 \\
\hline 65816 & $0.950 \pm 0.075$ & $\ldots$ & GROND & 0.17 \\
\hline 66252 & $0.960 \pm 0.075$ & $\ldots$ & GROND & 0.19 \\
\hline 66688 & $0.950 \pm 0.074$ & $\cdots$ & GROND & 0.11 \\
\hline 67127 & $0.936 \pm 0.074$ & $\cdots$ & GROND & 0.23 \\
\hline 67583 & $0.977 \pm 0.069$ & $\cdots$ & GROND & 0.30 \\
\hline 68017 & $1.016 \pm 0.066$ & $\ldots$ & GROND & 0.16 \\
\hline 68456 & $0.980 \pm 0.066$ & $\cdots$ & GROND & 0.54 \\
\hline 69196 & $1.017 \pm 0.057$ & $\cdots$ & GROND & 0.77 \\
\hline 69997 & $0.998 \pm 0.064$ & $\cdots$ & GROND & 0.25 \\
\hline 70850 & $0.977 \pm 0.062$ & $\ldots$ & GROND & 0.77 \\
\hline 71596 & $0.995 \pm 0.063$ & $\cdots$ & GROND & 0.72 \\
\hline 72379 & $1.009 \pm 0.063$ & $\cdots$ & GROND & 1.03 \\
\hline 73164 & $1.082 \pm 0.057$ & $\cdots$ & GROND & 0.27 \\
\hline 73959 & $1.038 \pm 0.056$ & $\ldots$ & GROND & 1.31 \\
\hline 74759 & $1.034 \pm 0.055$ & $\ldots$ & GROND & 0.51 \\
\hline 75557 & $1.016 \pm 0.067$ & $\ldots$ & GROND & 0.32 \\
\hline 76349 & $1.077 \pm 0.057$ & $\ldots$ & GROND & 0.25 \\
\hline 77141 & $1.117 \pm 0.058$ & $\cdots$ & GROND & 0.50 \\
\hline 77953 & $1.123 \pm 0.055$ & $\cdots$ & GROND & 0.86 \\
\hline 78755 & $1.123 \pm 0.062$ & $\ldots$ & GROND & 0.21 \\
\hline 79557 & $1.140 \pm 0.060$ & $\ldots$ & GROND & 0.39 \\
\hline 80358 & $1.199 \pm 0.052$ & $\cdots$ & GROND & 0.77 \\
\hline 81167 & $1.169 \pm 0.052$ & $\ldots$ & GROND & 0.84 \\
\hline 81946 & $1.149 \pm 0.058$ & $\ldots$ & GROND & 1.05 \\
\hline $125931-283858$ & $1.054 \pm 0.058$ & $0.121 \pm 0.036$ & UVOT+GROND & 1.08 \\
\hline $125931-283858$ & $1.241 \pm 0.022$ & $\ldots$ & UVOT+GROND & 2.33 \\
\hline 151489 & $1.240 \pm 0.044$ & $\cdots$ & GROND & 0.21 \\
\hline 155908 & $1.183 \pm 0.049$ & $\ldots$ & GROND & 0.76 \\
\hline 160328 & $1.182 \pm 0.044$ & $\ldots$ & GROND & 0.10 \\
\hline 164699 & $1.224 \pm 0.059$ & $\ldots$ & GROND & 0.23 \\
\hline 239811 & $1.158 \pm 0.058$ & $\cdots$ & GROND & 0.84 \\
\hline 250946 & $1.154 \pm 0.063$ & $\cdots$ & GROND & 0.53 \\
\hline $312320-474249$ & $1.005 \pm 0.248$ & $0.144 \pm 0.140$ & UVOT+GROND & 0.31 \\
\hline $312320-474249$ & $1.248 \pm 0.083$ & $\cdots$ & UVOT+GROND & 0.39 \\
\hline 329172 & $1.185 \pm 0.056$ & $\ldots$ & GROND & 0.31 \\
\hline 415466 & $1.133 \pm 0.061$ & $\cdots$ & GROND & 0.12 \\
\hline $501081-1101930$ & $1.147 \pm 0.076$ & $\ldots$ & GROND & 0.20 \\
\hline 501081 & $1.138 \pm 0.048$ & $\ldots$ & GROND & 0.50 \\
\hline 588101 & $1.161 \pm 0.075$ & $\cdots$ & GROND & 0.20 \\
\hline 669176 & $1.141 \pm 0.098$ & $\cdots$ & GROND & 0.11 \\
\hline 843664 & $1.075 \pm 0.066$ & $\cdots$ & GROND & 0.04 \\
\hline 1101930 & $1.096 \pm 0.101$ & $\ldots$ & GROND & 0.88 \\
\hline
\end{tabular}

We have a total of 46 epochs of data, and fit each with a simple power-law as well as with dust models based on Milky Way, Large and Small Magellanic Cloud dust based on the parametrization of Pei (1992). The results for the simple powerlaw as well as those with additional UVOT (and others) data are given in Table 5, and are plotted in Fig. 5. We have already remarked (see also Stratta et al. 2013) on the red-to-blue evolution from the prompt emission to the pre-rebrightening afterglow. The value derived from the decaying UVOT afterglow is in excellent agreement with the GROND-derived values for the beginning of the plateau phase. For the GROND data, we find a clear spectral evolution during the first day, which was already evident from the fact that the temporal slopes were different for each filter (Table 3 ). We are able to linearly (in $\log (t)$-space) fit the evolution with a steep rising slope, $\alpha_{\beta, 1}=2.25 \pm 0.37$. Starting on the second day, the afterglow SED has reached a constant value of $\beta \approx 1.25$; fitting these data yields $\alpha_{\beta, 2}=-0.14 \pm 0.06$, which is only in agreement with no further evolution at the $2 \sigma$ level, therefore, some chromatic evolution may still be present but it is of low significance. Data beyond 21 days (epochs 


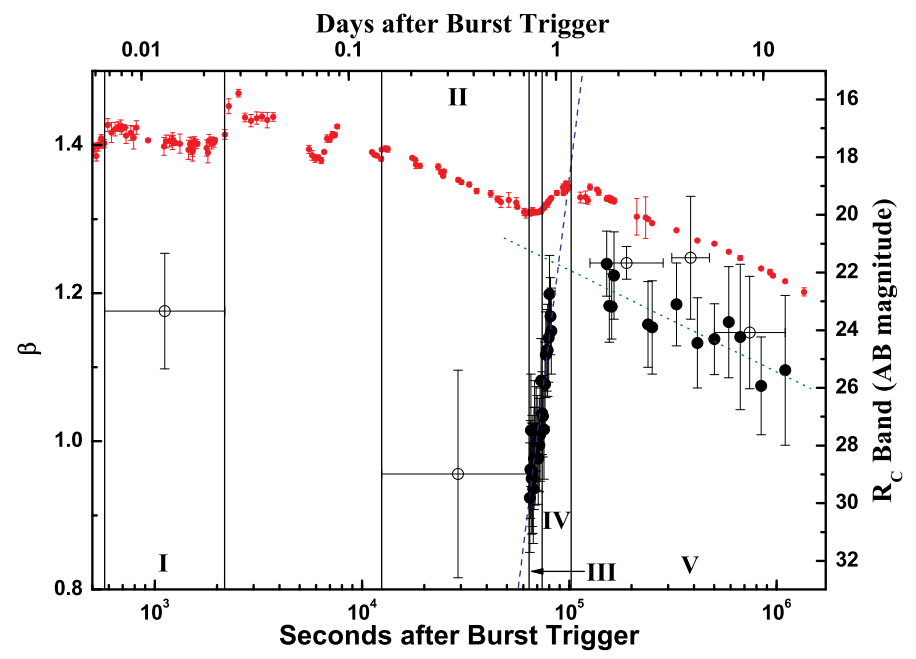

Fig. 5. Evolution of the spectral slope of the optical/NIR afterglow, $\beta$. Open points are either derived from UVOT (plus TAROT, REM in the first epoch) or from joint UVOT+GROND fits. Closed points are GROND-only. See Table 5 for the data. Red points are a composite $R_{C}$-band light curve of GRB 111209A (identical to the one given in Figs. 10 and 11), added for purposes of orientation. The regions are: I. Early prompt emission. II. First afterglow-like decay. III. Plateau. IV. Rebrightening. V. Late afterglow. The plateau/rebrightening shows a marked rise of the spectral slope, from $\approx 0.95$ to $\approx 1.25$, where it then remains essentially constant. The two different behaviours themselves can be fitted linearly (in temporal log-space), the rising phase has a slope of $2.25 \pm 0.37$ (dashed blue line), while the constant phase has a slope of $-0.14 \pm 0.06$ (dotted green line), indicating some low-significance chromatic evolution may still be present. Data beyond those shown here show a significant departure from a power-law form due to the increasing dominance of the supernova component.

41-46) are not displayed as the dominating supernova component (G15, K18A) induces a strong curvature into the spectrum, so power-law fits lose their validity. We furthermore perform three fits using both UVOT and GROND data, now derived from joint power-law fits to the three afterglow segments separated by the "steps" (Sect. 3.1.4, see also K18A); the time spans over which these fits have been performed are also given in Table 5. Our SED results are for the most part in good agreement with those given by Stratta et al. (2013); for example, they find $\beta=1.07 \pm 0.15$ using GROND data published in the GCNs (Kann et al. 2011a). Their spectral slope during the peak of the rebrightening, $\beta=1.0 \pm 0.1$, is somewhat bluer than what we expect from our $\beta$-evolution. Later SEDs, based only on UVOT data, show lots of scatter but agree within errors with our results.

The dust-model fits using GROND-only data yield discouraging results. At such low redshifts, it is not possible to distinguish between the three models using optical (and NIR) data only (Kann et al. 2006). Conversely, even small fluctuations in the $g^{\prime}$ data (which is especially susceptible to atmospheric effects during observations, such as residual twilight, seeing, and haze) lead to large differences in results, everything between moderate line-of-sight extinction of $A_{\mathrm{V}}=0.5 \mathrm{mag}$ to negative curvature. Furthermore, the simple power-law model fits are all already statistically acceptable (except those during the supernova phase as mentioned above). Stratta et al. (2013) reach similar conclusions. Our joint UVOT+GROND fits, though, yield the largest wavelength span possible, and improve upon the GROND-only analysis. While the last epoch yields no evidence for dust, and the middle one no statistically significant evidence, the first of the three joint fits (where UVOT data are densest and have the best $\mathrm{S} / \mathrm{N}$ ) reveals curvature in the SED in the observer-frame ultraviolet. Using the SMC dust model (LMC and MW dust yield similar results), we detect additional extinction along the line of sight at the $3.4 \sigma$ level: $A_{\mathrm{V}}=0.121 \pm 0.036 \mathrm{mag}$. This value is not in contradiction with the general result of low line-of-sight extinction, and a very typical value for GRB afterglows (e.g. Kann et al. 2006, 2010). This value was adopted in G15 and we also use it here where needed.

An important conclusion we can draw here is that we find no evidence for a thermally dominated spectrum such as in the case of the "Christmas Burst" GRB 101225A, which showed an evolving, cooling blackbody, and not a typical afterglow synchrotron spectrum (Thöne et al. 2011), nor do we see an extremely UV-bright blackbody spectrum as exhibited (probably) by GRB 110328A/Swift J164449.3+573451 (Levan et al. 2011) and (definitely) by Swift J2058.4+0516 (Cenko et al. 2012). This is a strong argument against GRB 111209A being similar to these classes of explosive transients.

\subsection{The host galaxy}

In the deep late-time data taken at 280 days post-trigger, we detect the host galaxy in $g^{\prime} r^{\prime} i^{\prime} z^{\prime}$ in the $3-5 \sigma$ range. While our $J H K$ observations reach deeper than any of our previous ones of this field, the host remains undetected. L14 also obtained host-galaxy observations, yielding clear detections in $g^{\prime} r^{\prime}$ which are in excellent agreement with the values we obtain (and have significantly higher $\mathrm{S} / \mathrm{N}$ ), an additional low-S/N detection in Hubble Space Telescope (HST) $F 336 W$ and Gemini $u^{\prime}$, as well as a much deeper limit in $J$ than the one we have achieved. The SN does not contribute significantly any more during this epoch, with expected magnitudes $g^{\prime} \approx 28.5 \mathrm{mag}, r^{\prime} \approx 28.0 \mathrm{mag}, i^{\prime} \approx 27.5 \mathrm{mag}$, $z^{\prime} \approx 27.2$ mag. We find that the host galaxy is an unresolved point source, and therefore must be very compact. This is confirmed by L14 who detect only marginal extension even in their highresolution HST imaging. We add their data to our host-galaxy study, using the host-galaxy magnitude they derive in $F 336 \mathrm{~W}$, just as for the afterglow+SN fit (G15,K18A).

We use the photometric SED-fitting code LePHARE (Arnouts et al. 1999; Ilbert et al. 2006) ${ }^{6}$ to determine hostgalaxy parameters from the detections. As a cross-check, in case there is still a contribution of the $\mathrm{SN}$, we also use the host-galaxy values as determined by our joint afterglow $+\mathrm{SN}$ fit $(\mathrm{G} 15, \mathrm{~K} 18 \mathrm{~A})$; we find the results are consistent with the previous fit, within uncertainties. Our fit result is shown in Fig. 6. We find that the host galaxy is a low-mass galaxy with very low global extinction (using the Calzetti et al. 2000 starburst-galaxy extinction law) a moderate star-formation rate (SFR; which is in full agreement with that derived from emission line properties by Krühler et al. 2015) but a high specific star-formation rate (sSFR); for result values, see Table 6. Due to the lack of NIR data and the low $\mathrm{S} / \mathrm{N}$ of the optical data, parameters such as age, SFR, sSFR and mass are not well-determined. From the emission lines in their spectra, L14 derive a moderately high metallicity. From the same spectra, Krühler et al. (2015) derive a metallicity that is 0.3 dex lower, but in agreement within the large errors.

To determine the offset of the afterglow from the host galaxy, we use the $r^{\prime}$-band image taken at 5.8 days (see Fig. 1), which has the highest detection $\mathrm{S} / \mathrm{N}$. Using the result catalogue of this image as an astrometry catalogue, we determine a mapping uncertainty between the two epochs of 0 ".018. We estimate the purely statistical error of the afterglow/host localization via the

6 http://www. cfht.hawaii.edu/ arnouts/LEPHARE 


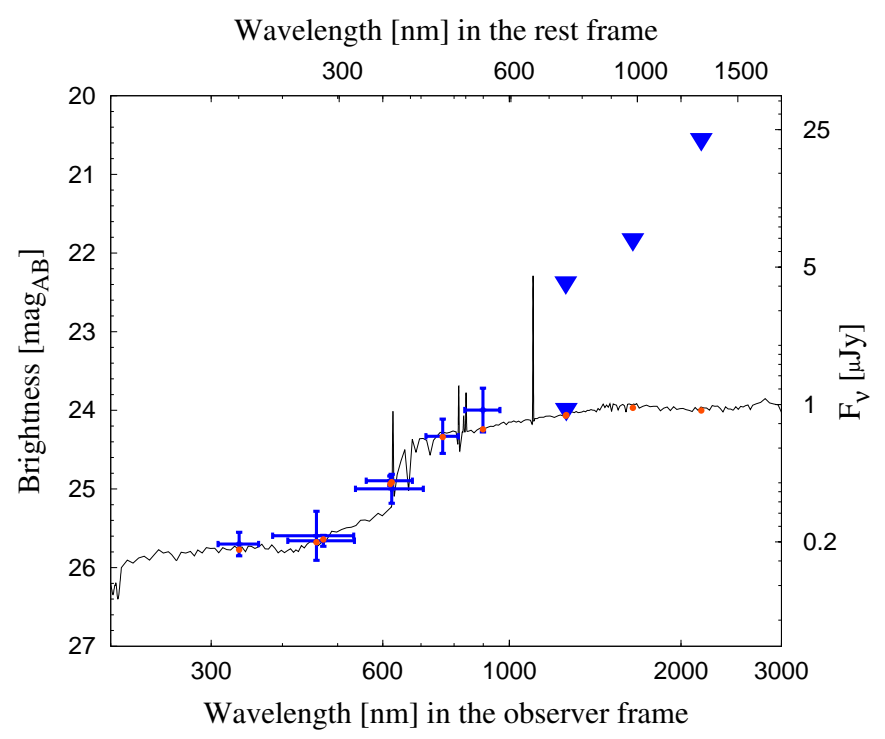

Fig. 6. LePHARE fit to the magnitudes of the GRB 111209A host galaxy. The detections are $F 336 W g^{\prime} g_{G}^{\prime} r^{\prime} r_{G}^{\prime} i_{G}^{\prime} z_{G}^{\prime}$, the upper limits $J J_{G} H_{G} K_{S, G}$ (the subscript $\mathrm{G}$ denotes GROND filters presented in this work, the non-GROND data have been taken from L14). The red $\bullet$ marks represent the photometry values as determined by the synthetic SED. The fit is acceptable, $\chi^{2}=1.36$. The host galaxy is very typical for a GRB host, a low-mass, low-extinction, young star-forming galaxy (see, e.g. Schulze et al. 2014 for a comparison). For specific values, see Table 6.

Table 6. Fit results for the LePHARE host-galaxy fit.

\begin{tabular}{lr}
\hline \hline Parameter & Value \\
\hline Redshift & 0.677 (fixed) \\
$\chi^{2}$ & 1.36 \\
Extinction law & Calzetti \\
$E_{(B-V)}$ & 0.04 \\
$M_{B}$ & -17.93 \\
Age $(\mathrm{Gyr})$ & $0.84_{-0.50}^{+1.24}$ \\
SFR $\left(M_{\odot} \mathrm{yr}^{-1}\right)$ & $0.22_{-0.01}^{+1.33}$ \\
$\log \mathrm{sSFR}(1 / \mathrm{Gyr})$ & $0.39_{-0.38}^{+2.52}$ \\
$\log \left(\operatorname{Mass}\left(M_{\odot}\right)\right)$ & $8.89_{-0.36}^{+0.58}$ \\
\hline
\end{tabular}

size of the mean stellar full-width at half maximum (FWHM) multiplied by the error of the afterglow/host (the afterglow, but also the host, are point sources), finding 0.011 for the afterglow and 0 ."176 for the host galaxy; the latter error dominates the total error on the offset 0.177 . We determine an offset of 0 ."396 in RA and -0 ."180 in Dec, and therefore, in total, an offset of 0 ". $44 \pm 0$ ". 18 . This translates to a projected offset of $3.06 \pm 1.25 \mathrm{kpc}$. This is somewhat larger than the median projected offset found by Bloom et al. $(2002,1.3 \mathrm{kpc})^{7}$, but still well within the distribution those authors found, which extends to $7 \mathrm{kpc}$ (we caution that this sample is not unbiased and may therefore not represent the true offset distribution).

L14 use their two epochs of HST WFC3 F336W data to determine the offset using a method analogous to our own. They derive a significantly smaller offset $\left(00^{\prime \prime} 011 \pm 00^{\prime \prime} .038\right)$, indistin-

\footnotetext{
7 We note that these authors used a different world model than we do, but as they themselves point out, the angular distance scale over a large redshift range is quite insensitive to redshift, and therefore also to small changes in the world model.
}

guishable from being right in the centre of the galaxy. As the HST data have both a better $\mathrm{S} / \mathrm{N}$ than our data and a much finer resolution, their offset value is likely more trustworthy than the one we have determined.

In passing, we note that G15 also derived the offset between the afterglow position and the SN position, and found that it was $<200$ pc (0 within errors), making it extremely unlikely that SN $2011 \mathrm{kl}$ is not caused by the same progenitor as GRB $111209 \mathrm{~A}$ itself.

\subsection{The light curve of GRB 130925A}

Another ULGRB with detailed GROND observations is GRB 130925A (see Appendix B for more details on this event). Greiner et al. (2014) have presented optical/NIR observations obtained with GROND and VLT/HAWK-I (late host-galaxy detections). These data show strong, rapid variability during the very long prompt emission phase. Furthermore, the afterglow is very strongly extinguished, the largest extinction measured so far with high significance along a line-of-sight to an afterglow. This leads to the optical transient not being detected at all in $g^{\prime} r^{\prime}$, whereas in $i^{\prime} z^{\prime}$, only the early optical emission of the prompt peak is detected above host-galaxy level. In $J H K_{S}$, both the early prompt emission as well as a late-time afterglow are detected. Greiner et al. (2014) present two SED fits of the optical NIR data. At peak, they measure an intrinsic spectral slope $\beta=1.3 \pm 0.4$ coupled with an extinction $A_{\mathrm{V}}=5.0 \pm 0.7$. The later afterglow, in combination with X-ray data, yields $\beta=0.32 \pm 0.03$ and $A_{\mathrm{V}}=6.56 \pm 0.76$. Both of these measurements must be treated with caution. The early optical/NIR emission is likely coupled to the largest prompt emission peak at high energies, and therefore is likely to have a different colour than the late afterglow. Additionally, the intrinsic SED may not be described by a simple power-law, though of course such a deviating intrinsic spectrum cannot be deduced from the data. The late SED is not based on contemporaneous data in the optical/NIR and the X-rays. Furthermore, the X-ray afterglow clearly stems from a different source than the optical/NIR emission (see Evans et al. 2014; Zhao \& Shao 2014; Piro et al. 2014, for interpretations). We therefore use both sets of values, as the true slope and extinction are likely to lie somewhere in the range.

Using our knowledge of the redshift $z=0.347$, the spectral slope and the extinction, we can use the method of Kann et al. (2006) and shift the light curve ${ }^{8}$ of GRB 130925A to $z=1$. For the SMC dust model (as used by Greiner et al. 2014), we find large corrections $d R c$ to $z=1$, despite the very low redshift. The "prompt" values yield $d R c=-2.91_{-1.04}^{+1.03} \mathrm{mag}$, whereas the "afterglow" values give $d R c=-5.29_{-0.94}^{+0.95} \mathrm{mag}$. The very large error bars are due to the very large - in absolute terms - errors of the line-of-sight extinction. The two results are discrepant at the $1.7 \sigma$ level. As stated above, the optical transient is not detected at all in the $r^{\prime}$ band. Since the method of Kann et al. (2006) is normalized to the $R_{C}$ band, we need to evaluate how the afterglow would look in the observed $r^{\prime}$ band. We subtract the individual host-galaxy magnitudes from all bands, and then create an $i^{\prime} z^{\prime} J H K_{S}$ SED at the prompt emission peak. We find results that agree within errors with those presented in Greiner et al. (2014). It is not possible to distinguish the dust model for such a low redshift (Kann et al. 2006), but Milky Waytype dust yields the smallest intrinsic spectral slope coupled with

\footnotetext{
8 More specifically, the light curve is given in the observer frame, but plotted as if the GRB had occurred at $z=1$.
} 


\section{A. Kann et al.: Complex GRB 111209A afterglow}

the highest extinction. Since several highly extinguished GRB afterglows have shown the presence of a prominent $\approx 2200 \AA$ bump (Krühler et al. 2008; Elíasdóttir et al. 2009; Perley et al. 2011; Zafar et al. 2012), it is quite likely that such a feature also exists in the spectrum of GRB 130925A, but the combination of low redshift, extremely high extinction, and a bright host galaxy makes it undetectable.

The extrapolation of the extremely red $\left(\beta_{0}=4.77 \pm 0.11\right)$ and curved SED at peak allows us to determine that the expected observed peak magnitude in the $r^{\prime}$ band would be $r^{\prime} \approx 23$ mag. We then take the (host-galaxy subtracted) $H$ band (to which we add $118 \mathrm{~s}$, to make $t_{0}$ identical with the trigger time of Fermi GBM), which offers the highest data density and latest detections, and shift it downward by the difference, a total of seven magnitudes (in Vega). This light curve is then corrected using the two $d R c$ values derived above. If one adds the large, one-magnitude error margin, the "afterglow value"-derived shifted light curve reaches the same luminosity as the corrected afterglow of GRB 111209A (Sect. 4.3). The "prompt value"-derived result is among the least luminous afterglows detected so far. This may indicate that the "afterglow value"-derived shift depicts a more realistic scenario, but there is no clear way to determine where within the range of $\approx 4$ magnitudes the shifted light curve truly lies.

\section{Discussion}

\subsection{Energetics}

The true energy release of GRB 111209A is an important parameter both for the determination of the nature of the event, as well as for the modelling of the event with spin-down emission from a magnetar.

Building upon the correlation between intrinsic peak energy of the prompt emission $E_{p, z}$ and isotropic-equivalent energy release $E_{\text {iso }}$ (Amati et al. 2002), Ghirlanda et al. (2004) discovered a tighter correlation between $E_{p, z}$ and $E_{\gamma}$, the collimationcorrected energy release (corrected for beaming; see also Friedman \& Bloom 2005). The "price" for a tighter correlation is the addition of a new parameter, the jet-break time $t_{\mathrm{b}}$, which is needed to determine the jet opening angle $\theta$ (Sari et al. 1999). Additionally, the external medium density $n_{\text {ISM }}$ (or the wind parameter $A_{\star}$, see, e.g. Zeh et al. 2006; Nava et al. 2006; Ghirlanda et al. 2006) and the efficiency $\eta_{\gamma}$ are needed as input parameters; these are usually best-estimates, with $n \approx 1-10 \mathrm{~cm}^{-3}$. Using the prompt energetics as derived by Golenetskii et al. (2011), we find $E_{p, z}=520 \pm 89 \mathrm{keV}$, and a bolometric (restframe $1-10000 \mathrm{keV}$ ) $\log E_{\text {iso,bol }}=53.83 \pm 0.06$ (one of the most energetic GRBs at $z<0.9$, Perley et al. 2014). GRB 111209A agrees well with the Amati correlation.

Our measurement of a jet-break time (G15,K18A, see Sect. 3.1.5) also allows us to place this GRB in the context of the Ghirlanda relation. Using the standard density values, we derive an opening angle of $\theta=0.15-0.19$ radian (for $n_{\mathrm{ISM}}=1 \cdots 10 \mathrm{~cm}^{-3}$ ). For $n_{\mathrm{ISM}}=0.1 \mathrm{~cm}^{-3}$ (as used by Stratta et al. 2013), we find $\theta=0.11$ radian, in good agreement with the value Yu et al. (2015) employed, but significantly less than found by Stratta et al. (2013). Both results are smaller than the value Ryan et al. (2015) derived from the X-ray afterglow, $\theta=0.34_{-0.13}^{+0.11}$ radian, but larger than the value. Metzger et al. (2015) derive, $\theta=0.05$ radian. We point out, however, that Metzger et al. (2015) overestimate the mean isotropic gammaray luminosity by a factor of ten; using input values from G15, it is $L_{\gamma} \approx 4 \times 10^{49} \mathrm{erg} \mathrm{s}^{-1}$. Using the correct value and the equation given in Metzger et al. (2015), we find $\theta \approx 0.16$ radian, in perfect agreement with our result above. We note that the range we find corresponds to an opening angle of 8.6-10.9 degrees. Prajs et al. (2017) use a mean opening angle of 12 degrees to estimate that the ULGRB rate is roughly similar to the superluminoussupernova (SLSN) rate they derive, and that SLSNe may therefore be associated with ULGRBs in a more general manner (see K18A for more discussion on ULGRBs and SLSNe). They use an estimate as " $[\mathrm{t}] \mathrm{o}$ date, jet-breaks have not been observed in ULGRBs" (Prajs et al. 2017). The upper bound we derive for the opening angle assuming a typical ISM density agrees well with their estimate, implying that their rate estimation does not need to be significantly changed. Perna et al. (2018), who study numerically whether or not BSG progenitors with fallback are able to launch jets and power ULGRBs, use an injection opening angle of $\theta=0.28$ radian, but note that the cocoon of the massive envelope will collimate the jet further, implying qualitative agreement with our opening angle result.

The main result, though, is that for all these values, GRB 111209A is a strong outlier of the Ghirlanda correlation and a hyper-energetic GRB, with $\log E_{\gamma}[\mathrm{erg}]=51.60-52.10$, a value similar to the hyper-energetic events studied by Cenko et al. (2011). To bring this GRB into agreement with the Ghirlanda relation ${ }^{9}$, assuming standard values otherwise, a significantly lower circumburst medium density, $n_{\mathrm{ISM}} \approx$ $10^{-4} \mathrm{~cm}^{-3}$, would be needed, leading to an opening angle of just $\theta=0.046$ radian (2.6 degrees, in this case $\log E_{\gamma}[\mathrm{erg}]=50.86$ ). Such a small opening angle would make it an outlier in the large sample of Ryan et al. (2015), who find $\theta \geq 0.055$ radian at $95 \%$ confidence, but in full agreement with the recent results of Wang et al. (2018), who find many early breaks ${ }^{10}$ and a typical $\theta=(2.5 \pm 1.0)^{\circ}$. We note here such a low circumburst density would be in agreement with the explanation Evans et al. (2014) propose for GRB 130925A (see K18A for more discussion) and that such low circumburst densities are not unprecedented, having been found for other GRBs from reverse-shock-flare modelling (Laskar et al. 2013, 2016; Alexander et al. 2017).

Gompertz \& Fruchter (2017, henceforth GF17) study GRB 111209A/SN 2011kl in the context of the magnetar model under the assumption that the magnetar powers the entire event, including the prompt emission and the afterglow. They present two separate lines of evidence that GRB 111209A is surrounded by a high circumburst density medium (in contrast to our argument using the Ghirlanda relation given above), and combined with a lower limit on the jet-break time, they conclude only an extreme magnetar rotating near break-up speed can power the event. This result is in contrast to other results, e.g. Yu et al. (2017) find the lowest rotation rate for SN $2011 \mathrm{kl}$ compared with a sample of SLSNe, but as GF17 point out, these studies only deal with powering the $\mathrm{SN}$ - see also the recent results from Wang et al. (2017), who use the bolometric light curve of K18A to derive magnetar-spin-down parameters. The rotation rate they find is slower than any in the SLSN-sample of Nicholl et al. (2017).

We find some inconsistencies in the work of GF17 that imply that a less extreme magnetar may also account for

9 Of course, it must be noted that the Ghirlanda relation is purely empirical, and so far has no clear physical meaning; therefore, being an outlier of this relation does not necessarily imply that any input parameters need to be adjusted to bring it into agreement with the relation. Recent research suggests it is less tight than once assumed (Wang et al. 2018).

${ }^{10}$ In these cases, of course, the narrow opening angles occur in typical external medium densities, whereas for GRB 111209A, the break is late but the density extremely low. 


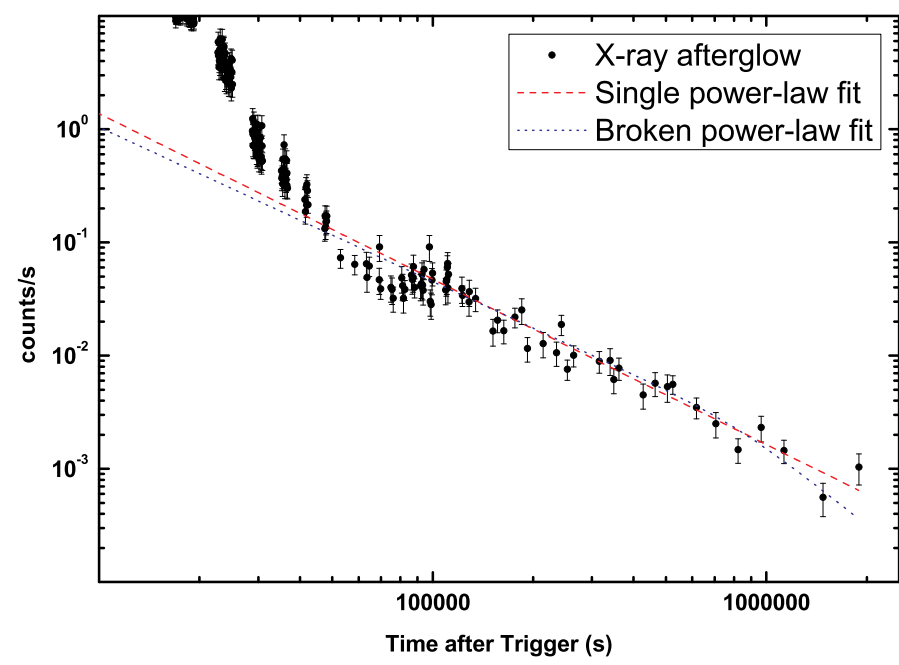

Fig. 7. Late X-ray afterglow of GRB 111209A. We show two fits using all data from 109 ks onward, a singe power-law fit (red dashed) and a broken power-law fit (blue dotted), with the parameters fixed to those derived from the optical afterglow fitting. See text for more details.

GRB 111209A. As a first note, a part of their work remarks upon the model of Metzger et al. (2015) and that work's strongly collimated jet, but as we pointed out above, Metzger et al. (2015) overestimate the isotropic luminosity of the GRB and therefore the collimation, implying that the modelling of Metzger et al. (2015) does not need to invoke extreme collimation. Alternatively, in the case of such collimation, the extremely low maximum density limit given by Metzger et al. (2015; which GF17 contrast with their significantly higher lower limit) is not valid anymore and the two results are not in conflict.

The second issue is the jet-break time. For one, GF17 completely ignore the optical-afterglow fit we presented already in G15, where we derive a jet break at 9.1 days (observer frame; 5.4 days rest-frame). They instead argue that the X-ray afterglow light curve (as given on the Swift XRT repository; Evans et al. 2007, 2009) shows no signs of a break until 22 days (observer frame) post-trigger. To put this statement in a statistical context, we fit the X-ray light curve. We use data starting 1.26 days post-burst; our first fit is with a single power-law, and our second fit is with a broken power-law, whereby we fix the jetbreak time and the post-break slope to the values derived from the optical fit. We fix the sharpness of the break $n=5$. These fits can be directly compared as they have identical degrees of freedom. For the first fit, we find $\alpha_{X, 1}=1.46 \pm 0.05$, and $\chi^{2}=41$ for 36 degrees of freedom; for the second fit, $\alpha_{X, 1}=1.36 \pm 0.06$ and $\chi^{2}=52$ (Fig. 7). De Pasquale et al. (2016) use a similar approach to derive a lower limit on the possible jet-break time of GRB 130427A, taking $\Delta \chi^{2}=2.7$ as a significance criterion. With $\Delta \chi^{2}=11$ in our case, the $\mathrm{X}$-ray afterglow alone significantly rules out a break at the optical time. We note, though, that the last XRT data point lies above the best fit, whereas the next-tolast data point at 17 days lies beneath the fit.

The final X-ray detection by Swift may have an alternate explanation. Levan et al. (2013) reported the detection of highly luminous X-ray emission associated with the SLSN SCP06F6, and Margutti et al. (2017) present not only limits on SLSNe and some further (faint) detections, but also compare them to the X-ray light curve of GRB 111209A. Their Fig. 1 shows that while most limits are deeper than the luminosity of the final data point of GRB 111209A $\left(\approx 9 \times 10^{43} \mathrm{erg} \mathrm{s}^{-1}\right.$ according to Margutti et al. $2017, \approx 6 \times 10^{44} \mathrm{erg} \mathrm{s}^{-1}$ following GF17), this last detection is significantly less luminous than the detection of SCP06F6. While no definite statement can be made, it is not implausible that SN $2011 \mathrm{kl}$ was accompanied by luminous $\mathrm{X}$-ray radiation, which we are detecting here. We refer the reader to K18A and references therein for multiple lines of argument as to why SN 2011kl resembles SLSNe more than it does typical GRB-SNe. The nature of luminous X-ray radiation accompanying SLSNe is far from being clear, as is the evolution of such $\mathrm{X}$-ray light curves compared to their optical counterparts. The UV/optical evolution of SCP06F6 was significantly slower than that of the less luminous SN 2011kl. Therefore, it may be possible that an X-ray transient associated with SN 2011kl itself (and not the GRB 111209A afterglow) would also evolve faster than the X-ray transient associated with SCP06F6, and be detectable by Swift at the given time. Therefore, the late X-ray light curve could consist of a breaking afterglow caused by an achromatic jet break and a rising (possibly peaking, as the detection is near the SN light-curve peak) SN X-ray component.

Bearing this in mind, we repeat the exercise and fit the X-ray light curve again, excluding the very last detection, and now find $\alpha_{X}=1.49 \pm 0.06$, and $\chi^{2}=39$ for 35 degrees of freedom, $\alpha_{X}=1.41 \pm 0.06$ and $\chi^{2}=40$, respectively; it is now $\Delta \chi^{2}=1$, implying the X-ray data (after exclusion of the last pojnt) do not strongly support a jet break but certainly do not rule it out either. A free fit to the entire data (after 1.26 days) does not find a break, whereas the exclusion of the last point leads to a break being found, but the break-time and post-break slope are degenerate, anchored by only a single data point (the last one in this fit, and the next-to-last one in total).

A further issue involves the broadband SED of the GRB, which GF17 argue is one of the indicators of a high circumburst density. We note that they use optical/NIR afterglow data from G15 to derive the SED in this regime. The values of $\beta_{o}$ and $A_{\mathrm{V}}$ they derive are perfectly consistent with our results. Furthermore, they derive a significantly steeper slope in the X-ray regime, which we confirm by using time-sliced spectra (from $109 \mathrm{ks}$ onward, beyond the plateau phase, Gendre et al. 2013) from the XRT repository. We derive a spectral slope difference of $\Delta \beta_{X, o}=0.43 \pm 0.19$, which would be indicative of a cooling break between the optical and $\mathrm{X}$-ray regimes, as also stated in GF17. The problem arises from the value of the decay slope of the optical/NIR afterglow. GF17 use nine epochs of GROND data unaffected by SN 2011kl to derive a slope $\alpha_{o}=1.30 \pm 0.05$. This slope is shallower than the one we derive, $\alpha_{o}=1.55 \pm 0.01$. While GF17 do not show their fit or give a goodness-of-fit value, the value they derive points to them not taking the rebrightening episodes into account. Using their value and comparing it with the X-ray decay slope, they find $\Delta \alpha_{X, o} \approx 0.25$, as expected for a cooling break in the case of a constant-density medium and slow cooling (e.g. Zhang \& Mészáros 2004). Our measured X-ray decay slope, especially if a single power-law fit is assumed (as GF17 do), lies close to our measured optical decay slopes, which is unexpected in the case of a cooling break lying between the X-ray and the optical domain, unless the circumburst medium density $\propto \rho^{-1}$ (between a constant-density and a wind medium) and therefore $v_{c}$ does not evolve and $\alpha_{o}=\alpha_{X}$. To further study this aspect, we create three NIR-to-X-ray SEDs, following the SED epochs given in Table 5, that is, for each of the smoothly decaying segments of the late afterglow between the "steps". These SEDs are shown in Fig. 8. We fit them with single power-laws, broken power-laws, and broken power-laws with $\beta_{2}=\beta_{1}+0.5$. We find no significant evidence that the SEDs cannot be explained by single power-laws; in all cases where a broken power-law is involved, the break is found in the range $0.5-2 \mathrm{keV}$, within the X-ray data itself. This short, steeper segment yields a 


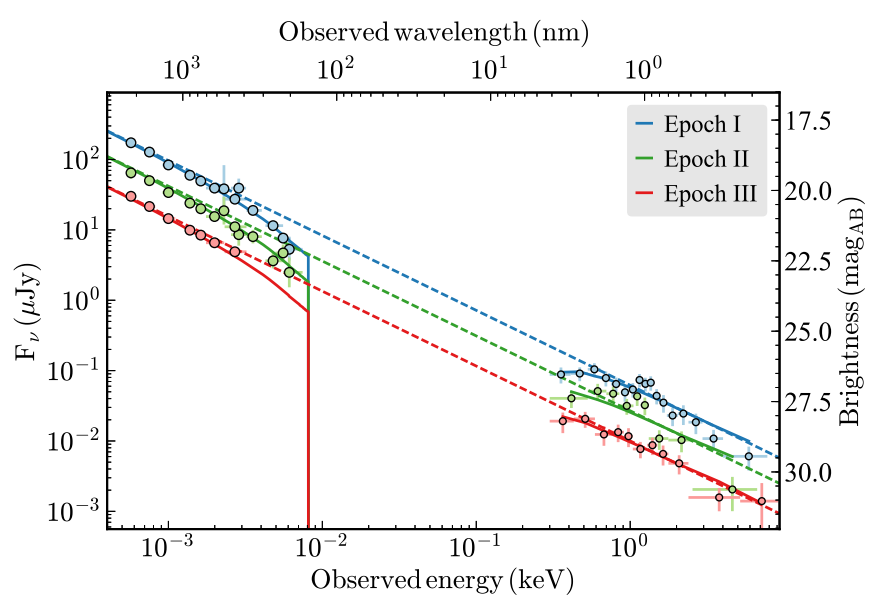

Fig. 8. Optical-to-X-ray SED fits for the late afterglow. The three epochs are the ones given in Table 5, between the "steps". The SEDs are all well-fit with single power-laws, and the derived parameters are identical in all three cases. There is no significant evidence for spectral evolution or the existence of a cooling break.

slightly improved fit, but in all cases, these improvements are not statistically significant. The spectral slope for all three fits is identical and in excellent agreement with the intrinsic spectral slope found in the optical/NIR SED alone; the same is true for $A_{\mathrm{V}}$, we give the values in Table 7. All in all, we find no significant evidence for a cooling break or spectral evolution.

To further study the likely location of the cooling break, we use the $\alpha-\beta$ relations. We only look at the simple models (e.g. Zhang \& Mészáros 2004, their Table 1, see also Gao et al. 2013) and find fast cooling is ruled out in all cases. The intrinsic spectral slope we derive, $\beta=1.06$, is relatively red and would usually be indicative of the regime blueward of the the cooling break $v_{c}$, and an electron distribution power-law slope $p=2.12$, and implied slope redward $\beta_{\mathrm{IR}}=\beta_{o, X}-0.5=0.56$ which is in perfect agreement with the mean value found in Kann et al. (2006). But taking the rather steep afterglow decay slope (as mentioned, $\alpha_{o}=\alpha_{X}$ within small errors) into account, we find the only $\alpha-\beta$ relation in full agreement with the data (i.e. $=0$ within errors) is for slow cooling, spherical expansion, a constant-density medium ("ISM"; note that GF17 consider an ISM medium ruled out in the case of the narrow-jet solution of Metzger et al. 2015), and a cooling break blueward of the X-ray regime (therefore, the marginal curvature seen in the harder $\mathrm{X}$-rays may be induced by the curvature of the cooling break). This directly implies a very soft electron distribution powerlaw index of $p=3.12$, a rare case, but not unheard of and in agreement with the non-universality of $p$ in GRB afterglows (Shen et al. 2006). This result therefore does not support the claim of GF17 concerning a high circumburst density, as $v_{c}$ scales with $n^{-1}$ (e.g. Zhang et al. 2007). We note that if we were to assume a higher column density (GF17 derive lower limits in the range $10 \ldots 10^{4} \mathrm{~cm}^{-3}$ from the radio observations, see below), it implies that the event is an even stronger outlier of the Ghirlanda relation, and even more energetic; for example, all other parameters unchanged, for $n=100 \mathrm{~cm}^{-3}$, we find an opening angle $\theta \approx 0.26$ radian (14.9 degrees) and a collimationcorrected energy release of $\log E_{\gamma}[\mathrm{erg}]=52.4$.

In the simple models we look at, this also implies $p=\alpha_{\text {post-break }}$, which would imply $\alpha_{\text {post-break }}>3$, significantly steeper than the value $\alpha_{\text {post-break }}=2.33 \pm 0.17$ which we find. It is therefore possible that there is indeed a contribution of SN $2011 \mathrm{kl}$ even in the $u$ band, and that the intrinsic afterglow decay is even steeper than what is observed when assuming SN $2011 \mathrm{kl}$ does not contribute in the $u$ band, which implies that SN $2011 \mathrm{kl}$ would be even more luminous than the result presented in G15 and K18A, further strengthening the claim that this $\mathrm{SN}$ is dissimilar to normal GRB-SNe.

We note that for high values of the energy fraction stored in magnetic fields $\epsilon_{B} \gtrsim 0.01 \ldots 0.1$, synchrotron cooling would become important, and $v_{c}$ would lie redward of the X-rays, and the X-ray flux would become independent of density. But for one, we find from the closure relations that the cooling break lies blueward of the X-rays. Furthermore, several works have performed afterglow modelling and have shown that typically $\epsilon_{B}$ is very low, likely $\gtrsim 10^{-4}$ (Kumar \& Barniol Duran 2010; Santana et al. 2014; Wang et al. 2015). These findings strengthen our result of a cooling break blueward of the X-rays.

Finally, GF17 use modelling of the radio data (Hancock et al. 2011, 2012) to derive further lower limits on the circumburst density, again finding moderately high values contrasting our estimation given above under the assumption that the Ghirlanda relation is valid. The spectral shape of the radio detections is peculiar, which GF17 explain by interstellar scintillation. Alternatively, the anomalous spectrum may be created by the overlap of multiple components; for example, a reverse shock and a forward shock, as in the cases of GRB 130427A (Laskar et al. 2013; Perley et al. 2014) and GRB 160625B (Alexander et al. 2017; Troja et al. 2017). In such a case, scintillation would not be necessary to fully explain the deviation from the expected spectrum (though of course a lower amount of scintillation may still exist), the source size could be significantly larger and again, the limits on the circumburst medium density could be lower. As there are no further X-ray or radio observations, to our knowledge, however, neither of our alternative explanations can be pursued any further.

Metzger et al. (2018) recently presented a study on the effects of fallback accretion on rapidly spinning proto-magnetars. They find that under certain conditions, the additional accretion can keep the magnetization within a certain regime which enables the launch of a relativistic jet for thousands of seconds, while at the same time also enabling additional energy injection into the latetime supernova, creating a viable solution to GRB 111209A/SN $2011 \mathrm{kl}$. One caveat of this model is that it does not imply that the maximum extracted energy from the magnetar increases compared to the case without any additional accretion.

The total rotational energy budget of a magnetar is

$E_{\mathrm{rot}}=\frac{I}{2} \Omega_{0}^{2}, \Omega_{0}=\frac{2 \pi}{P_{0}}$,

where $I \approx 1.3 \times 10^{45} \mathrm{~g} \mathrm{~cm}^{2}$ is the moment of inertia of the neutron star (assuming a typical mass $M_{\mathrm{NS}}=1.4 M_{\odot}$ ), $\Omega_{0}$ is the initial angular velocity and $P_{0}$ is the initial spin period. For a typical magnetar near break-up rotation speed $\left(P_{0} \approx 1 \mathrm{~ms}\right)$, it is $E_{\mathrm{rot}} \approx 3 \times 10^{52} \mathrm{erg}$, though Metzger et al. (2015) argue that neutron stars near the maximum possible mass (see Margalit \& Metzger 2017; Shibata et al. 2017; Ruiz et al. 2018; Rezzolla et al. 2018, for recent research) and with larger radii and extreme spin periods could have up to an order of magnitude higher $E_{\text {tot }}$. We note that GF17 argue that "the majority of this [energy] is carried away by neutrinos". Such a mechanism would be expected in Type II core-collapse SNe, but in the case of a spindown-powered central engine, such as a magnetar, the energy is released as a Poynting flux which is converted into radiation and kinetic energy. While neutrinos may be produced in the dissipation region via $p-\gamma$ interactions, the expected cross section and neutrino flux are very low (Zhang \& Kumar 2013). 
Table 7. Results of the optical-to-X-ray SED fits using a single power-law.

\begin{tabular}{lccccccc}
\hline \hline $\begin{array}{l}\text { Epoch } \\
\text { (time span in s) }\end{array}$ & $\begin{array}{c}\text { Fit statistic/d.o.f. Fit statistic/d.o.f. Fit statistic/d.o.f. } \\
\left(\chi^{2}, \text { optical) }\right.\end{array}$ & $\begin{array}{c}\text { Normalisation } \\
(\text { C-stat, X-rays) }\end{array}$ & $\begin{array}{c}\text { (Total) } \\
\left(10^{-5} \mathrm{keV} \mathrm{cm}^{-2} \mathrm{~s}^{-1} \mathrm{keV}^{-1}\right)\end{array}$ & $\begin{array}{c}\text { Spectral } \\
\text { slope } \beta\end{array}$ & $\begin{array}{c}A_{\mathrm{V}} \\
(\mathrm{mag})\end{array}$ & $\begin{array}{c}\mathrm{NH} \\
\left(10^{20} \mathrm{~cm}^{-2}\right)\end{array}$ \\
\hline I (125931-283858) & $11.53 / 13$ & $134.43 / 170$ & $145.96 / 179$ & 9.35 & $1.064_{-0.016}^{+0.017}$ & $0.122_{-0.023}^{+0.024}$ & $5.2_{-5.2}^{+6.0}$ \\
II (312320-474249) & $12.43 / 13$ & $84.83 / 85$ & $97.26 / 94$ & 4.05 & $1.059 \pm 0.031$ & $0.209_{-0.075}^{+0.085}$ & $1.8_{-1.8}^{+9.3}$ \\
III (501081-1101930) & $1.46 / 7$ & $76.32 / 105$ & $77.77 / 108$ & 1.51 & $1.060_{-0.028}^{+0.026}$ & $0.049_{-0.049}^{+0.087}$ & $0.1_{-0.1}^{+8.5}$ \\
\hline
\end{tabular}

Therefore a large fraction of the energy is available to drive the GRB, afterglow, and supernova.

Without broadband modelling, which is beyond the scope of this work (and may be hard to achieve considering how sparse the radio data is), it is not possible to pin down the true energetics of the entire event, but we can make an estimate. The collimation-corrected prompt energy release, as has been pointed out, varies strongly depending on the assumed circumburst medium density, from $\log E_{\gamma}[\mathrm{erg}]=50.9$ for the "Ghirlanda" solution, $\log E_{\gamma}$ [erg] = 51.6-52.1 for typical density values, and up to $\log E_{\gamma}[\mathrm{erg}] \approx 52.4$ for a density ten times higher than typical values, representative of the indications of high density that GF17 have derived.

The two further components that need to be taken into account are the kinetic energy of the $\mathrm{SN} E_{k, \mathrm{SN}}$ and the kinetic energy of the ultrarelativistic jet $E_{k, \text { Jet }}$. For the bolometric light curve presented in G15, these authors derive $E_{k, \mathrm{SN}}=(5.5 \pm 3.3) \times 10^{51} \mathrm{erg}$. We look to the work of Wang et al. (2017), who applied the magnetar model to the bolometric light curve of K18A, to derive estimates of $E_{k, \mathrm{SN}}$. We use the values they derive for models B1 and $\mathrm{C} 1$ (pure magnetar, and magnetar $+{ }^{56} \mathrm{Ni}$, respectively, both for opacity $\kappa=0.07 \mathrm{~cm}^{2} \mathrm{~g}^{-1}$ ) and $E_{k, \mathrm{SN}}=0.3 M_{\mathrm{ej}} v_{\mathrm{sc} 0}$, with $M_{\mathrm{ej}}$ the ejecta mass of the SN and $v_{\mathrm{sc} 0}$ the ejecta scale velocity, and find $E_{k, \mathrm{SN}}=\left(1.1_{-0.64}^{+0.72}\right) \times 10^{52} \mathrm{erg}$ and $E_{k, \mathrm{SN}}=\left(1.3_{-0.67}^{+0.72}\right) \times 10^{52} \mathrm{erg}$, respectively. We note here that depending on the asphericity of the SN explosion (which may occur along the polar axis as a result of the jet), this energy may be overestimated by a factor of two to five (Mazzali et al. 2014).

The broadest possible spread of energies concerns $E_{k, \text { Jet }}$. This value is connected to the energy released in gamma-rays via the efficiency $\eta_{\gamma} \equiv E_{\gamma \text {,iso }} /\left(E_{\gamma \text {,iso }}+E_{k \text {,iso }}\right)$, where $E_{k \text {,iso }}$ is the isotropic kinetic energy of the fireball at the end of the prompt emission (Zhang et al. 2007). Modelling of GRB prompt emission and afterglows has shown that $\eta_{\gamma}$ can have a wide range of values, for example, $\$ 50 \%$ (Beniamini et al. 2015), and even somewhere between $0.01 \%$ and $90 \%$ (Zhang et al. 2007; Wang et al. $2015)$, implying $E_{k \text {,iso }}=(0.1 \ldots 10000) E_{\gamma, \text { iso }}$. For very low $\epsilon_{B}$ values (see above), radiative efficiencies are typically on the or-

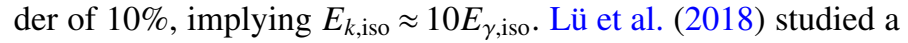
sample of GRB-SNe and, among other results, derived efficiencies for these events. They find that GRB 111209A is a strong outlier compared to the rest of the sample, with a very high efficiency of $76 \%$, implying that, in contrast to all other GRB/SNe they study, the GRB energy dominates compared to the SN energy. We note that this still depends on which circumburst medium density is assumed, as we have derived a broad range of potential collimation-corrected energy releases, which are coupled to $E_{k, \text { Jet }}$ via the efficiency $\eta_{\gamma}$. For our range of collimationcorrected $E_{\gamma}$ values, this implies $\log E_{k, \mathrm{Jet}} \approx 50.4 \ldots 51.9$.

In total summation, we find that, assuming, as pointed out above, neutrino losses are negligible, a "normal" magnetar would be able to power the entire event, reasonably independent of circumburst medium density, albeit with a very short rotation period, as GF17 imply (in full agreement with Lü et al. 2018 who find that GRB 111209A/SN 2011kl lies very close to the classical magnetar limit). Of the three components, $E_{k, \mathrm{SN}}$ has the least insecure value and sets a lower bound (assuming no asphericity) of the total energy of the event at $\approx 10^{52} \mathrm{erg}$ which is still within the bounds of the energy a classical magnetar can deliver. A more powerful magnetar (the "Metzger magnetar") could power the event while not rotating near break-up velocity. For a $10^{53}$ erg magnetar, even if the total energy of the event is equipartitioned between the kinetic energy of SN 2011kl and the energy of GRB 111209A and its jets, a spin period of $\approx 3 \mathrm{~ms}$ is derived. Such a high-mass neutron star near the NS/BH boundary would indeed be in agreement with a high initial spin period, as shown in the models of Aguilera-Dena et al. (2018). We therefore conclude that GRB 111209A/SN 2011kl can be powered by a magnetar central engine, and other energy sources (e.g. a Black-Hole central engine) that would be in conflict with the interpretation of the spectrum (G15, Mazzali et al. 2016) are not needed.

\subsection{Models for the plateau/rebrightening phase during Day 1}

We have found a remarkable behaviour during the first day of GROND observations. The afterglow, instead of fading, brightens slowly, then very steeply, and does so at a somewhat different rate in each band, leading to a rapid colour evolution seen especially well in the spectral slopes of the SEDs of each GROND epoch. Suffice to say, this is not typical behaviour for a GRB afterglow, and the simplest versions of the fireball model do not explain this evolution. We must therefore turn to more advanced scenarios.

\subsubsection{A powerful energy injection?}

Several GRBs have shown very strong rebrightenings at late times (i.e. hours to days, and therefore unrelated to the prompt emission), where the afterglow did not just show a flattening of its decay, but actually increased significantly, often by more than a magnitude. Examples for such events have been seen in, for example, GRB 970508 (Djorgovski et al. 1997; Castro-Tirado et al. 1998), GRB 030329 (Uemura et al. 2003; Lipkin et al. 2004), GRB 060206 (Woźniak et al. 2006; Monfardini et al. 2006), GRB 070125 (Updike et al. 2008; Chandra et al. 2008), GRB 080928 (Rossi et al. 2011), and GRB 090926A (Rau et al. 2010; Cenko et al. 2011); see also Laskar et al. (2015) for a recent comprehensive study of several further events. Yu et al. (2015) model the rebrightening (based on data from Stratta et al. 2013) with such an energy injection, and Zhang \& Mészáros (2001) even make the case that a millisecond magnetar engine would produce such a bump. On the other hand, such energy injections do not exhibit spectral changes (stemming from a single blastwave, with non-variable microphysical parameters, see especially Laskar et al. 2015), and therefore 


\section{A. Kann et al.: Complex GRB 111209A afterglow}

this interpretation seems unlikely - indeed, Zhang \& Mészáros (2001) specifically predict an achromatic bump.

\subsubsection{A two-component jet model?}

In recent years, GROND has revealed multiple complex afterglows which exhibit rebrightenings which often rise extremely rapidly and show colour changes across the rebrightening, such as GRB 080413B (Filgas et al. 2011b), GRB 081029 (Nardini et al. 2011), GRB 091029 (Filgas et al. 2012), GRB 100621A (Greiner et al. 2013) and GRB 100814A (Nardini et al. 2014). As with GRB 111209A, the simultaneous seven-colour capabilities of GROND allowed a detailed study of the SED evolution in those cases as well. GRB 081029 shows a similar evolution to GRB 111209A, with a steep rise in slope from $\beta \approx 0.77$ to $\approx 1.05$. Also, no contemporaneous rise was detected in the X-rays. GRB 080413B exhibits a switch from a very flat early $\operatorname{SED}(\beta=0.22)$, to a much steeper later one $(\beta \approx 0.9)$; and again there is no rebrightening seen in the X-rays ${ }^{11}$. GRB 100814 A is similar to GRB $080413 \mathrm{~B}$ with a transition from a once more very flat early $\operatorname{SED}(\beta \approx 0.10)$, to a much steeper later one $(\beta \approx 0.7)$. GRB 091029 shows more mild spectral evolution but a complete decoupling of optical and X-ray evolution. GRB 100621A shows very high line-of-sight extinction which complicates the determination of spectral changes, but features the steepest rise to peak of all these events. Additionally, GRBs 081029, 100621A and 100814A show superposed variability on top of the peak. Such a phenomenon cannot be checked for in GRB 111209A due to the lack of dense, high $\mathrm{S} / \mathrm{N}$ coverage during this time.

One explanation that may work in at least some of these cases is a two-component/double-jet model (e.g. Berger et al. 2003; Peng et al. 2005; Granot et al. 2006). In this scenario, the central engine emits a narrow, highly relativistic jet which is responsible for the prompt emission and the early afterglow, and a much wider and slower jet which produces a rebrightening when it decelerates, thereafter dominating the afterglow emission, especially in the radio regime. If the emission regions of these two jets have different microphysical parameters, their resulting spectral energy distributions will look different, causing a spectral change. Filgas et al. (2011b) successfully invoked this model to explain GRB 080413B. In the case of GRB 081029, Nardini et al. (2011) argued that the generic two-component jet model is unable to explain the very steep rebrightening, though it may work if one assumes that the wide jet was launched at a significantly later time (and the lower Lorentz factor may suppress high-energy emission, making it undetectable because the X-ray afterglow of the narrow jet outshines it). Even in the case of GRB 100814A, the decomposition into two overlapping components yields a steep rise of the second component (Nardini et al. 2014).

The extremely long duration of the prompt emission of GRB 111209A makes it a prime candidate for the launch of multiple jets at different $t_{0}$ times with different Lorentz factors. To check if it is possible to explain the temporal and spectral evolution of the afterglow of GRB 111209A with two afterglow components with different spectral slopes, we use a multi-band fitting procedure which is able to incorporate multiple broken power-laws as well as components of different colour (e.g. Perley et al. 2008a,b, 2014). The result is shown in Fig. 9. We find an acceptable $\left(\chi^{2}=319\right.$ for 285 degrees of freedom $)$ fit with decay slopes $\alpha_{1}=1.47 \pm 0.02$ and $\alpha_{2}=1.60 \pm 0.03$. The former value is

11 The actual rebrightening is missed in the optical/NIR as well due to lack of telescope coverage. steeper than we found by fitting the UVOT data alone, the latter value in perfect agreement with the GROND-only fit. The spectral slope changes by $\Delta \beta=0.39 \pm 0.03$, also in agreement with what we found by fitting the two data sets separately. What is astonishing is the rising slope of the second component; we find a value of $\alpha_{r}=-39 \pm 1$, which exceeds even the rise of GRB 100621A ( $\alpha_{r} \approx-14$, Greiner et al. 2013). Here, it should be mentioned that the $t_{0}$ that we use is the Swift trigger time, whereas the GRB clearly started several thousand seconds earlier. Setting $t_{0}$ to an earlier time, though, simply makes the slopes steeper; although, at close to one day after the GRB, such a change would have only a minimal influence. The situation therefore is similar to the findings of Huang et al. (2006) for GRB 030329, who were unable to model the rebrightening correctly as the data was too steep for their numerical model. Our fit, of course, is entirely empirical.

One aspect we have not incorporated is a "hidden" jet break of the narrow jet, which, for example, was needed to correctly fit the afterglows of GRBs 080413B and 100814A (Filgas et al. 2011b; Nardini et al. 2014). The combination of multiple small energy injections (see Sect. 4.2.3 below) and the rising SN at late times does not allow us to deduce such a hidden component, but it is very likely such a break would occur significantly earlier than the late break we do detect in the multi-band afterglow+SN fitting (K18A).

A detailed modelling, especially under the assumption of a strongly delayed $t_{0}$ and possibly in combination with a surrounding low-density void (as Evans et al. 2014 invoke to explain the properties of GRB 130925A), is beyond the scope of this paper, however. Here, we conclude that the rebrightening as well as the spectral slope change can in principle be accommodated by the superposition of two different afterglow components, without the need to invoke, for example, time-variable microphysical parameters (within a single emission region).

\subsubsection{Energy injections into the late afterglow}

Our light curve fits in Sect. 3.1.4 have revealed that after the purported peak and before the SN, the light curve did not decay entirely smoothly. Instead, it experienced two short flattening phases, before resuming a similar decay to beforehand. For the three sections, we find $\alpha_{3.1}=1.59 \pm 0.03, \alpha_{3.2}=1.44 \pm 0.09$ and, from the broken power-law $+\mathrm{SN}$ fit, $\alpha_{\text {late, } 1}=1.55 \pm 0.01$. These three decay slopes, while not strictly identical, are very similar to each other. Additionally, we detect no more significant colour changes during this phase (Sect. 3.2).

This combination of factors points to the variability being due to energy injections into the afterglow, exactly the explanation we can likely rule out for the main rebrightening. Such "refreshed shocks" can be due to slowmoving shells in the jet catching up with the forward-shock front, or late ejection of shells (e.g. Rees \& Meszaros 1998; Panaitescu et al. 1998; Kumar \& Piran 2000; Sari \& Mészáros 2000; Zhang \& Mészáros 2002) - especially the latter case is attractive in consideration of the extreme duration of GRB 111209A (Yu et al. 2015). Additional emissive components due to very long-lasting central engine activity (e.g. Dai \& Lu 1998; Rees \& Mészáros 2000; Ghisellini et al. 2007) are less likely to contribute, both due to the achromatic evolution (energy injections do not change the electron index $p$ or microphysical parameters, whereas a central-engine flare would likely exhibit a different spectrum), and the "step-like" afterglow form (a central-engine flare would be superposed, and the later afterglow would lie on the extrapolation of the earlier decay). 


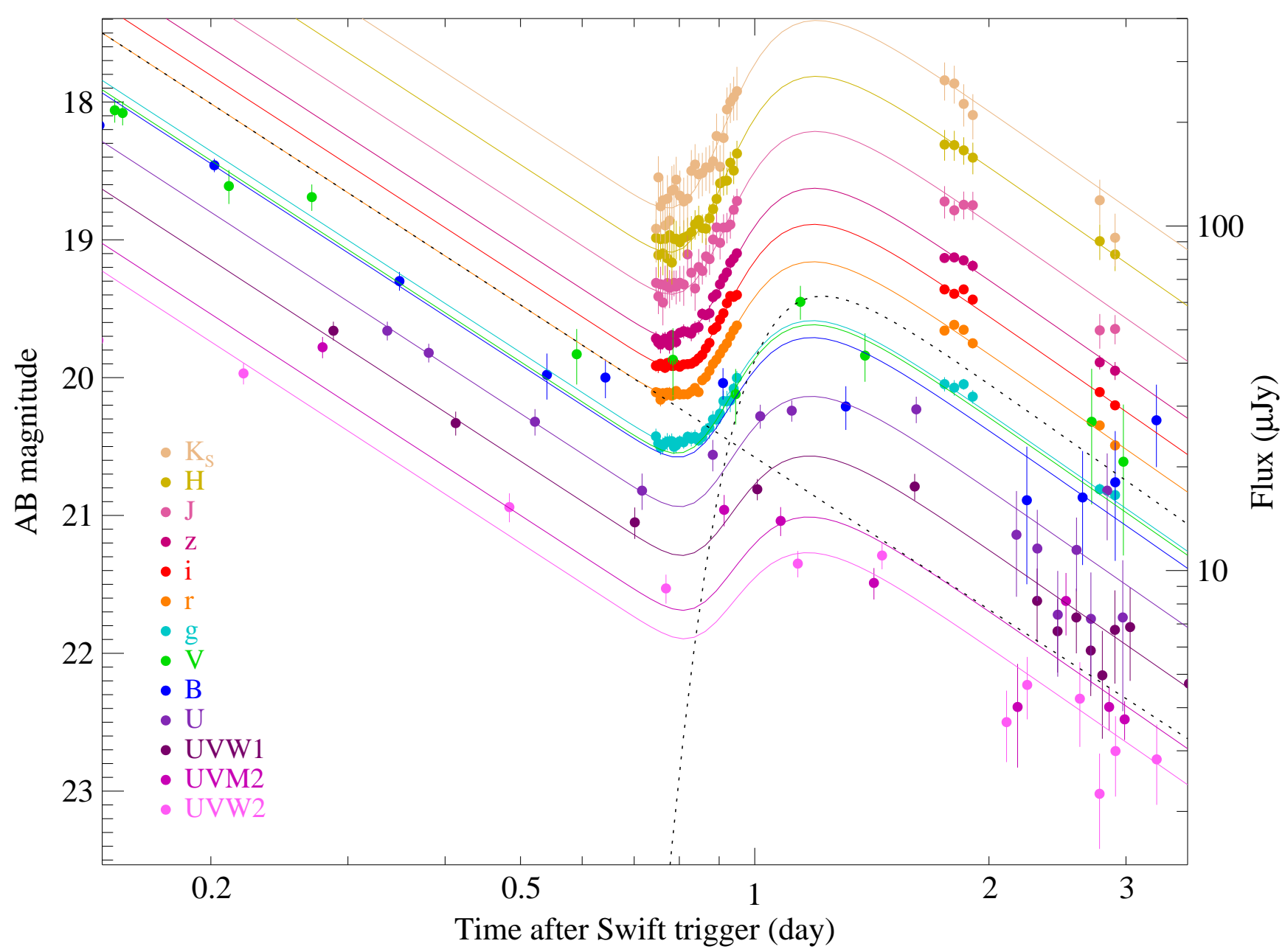

Fig. 9. Decomposition of the optical/NIR afterglow of GRB 111209A across the rebrightening episode. UVOT data are the only data available before $\approx 0.8$ days. With the beginning of GROND observations, a steeply rising second component becomes dominant (the two different components are shown as dotted lines for the $r^{\prime}$ band), leading to a colour change in the afterglow. The fit ends before the first "step" in the late afterglow.

Energy injections creating such "steps" have been proposed to explain similar features in such afterglows as GRB 030329 (e.g. Granot et al. 2003), GRB 021004 (Björnsson et al. 2004; de Ugarte Postigo et al. 2005) and GRB 060526 (Thöne et al. 2010). GRB 090926A at later times also exhibited a very similar behaviour (Rau et al. 2010; Cenko et al. 2011). They may also play a role in the light curves of SLSNe (Yu \& Li 2017), though the density variation model may also apply (Inserra et al. 2017).

\subsection{The luminosity of the afterglows of GRB 111209A and GRB 130925A}

In Kann et al. (2010), we presented a large sample of Swift-era long-GRB afterglow light curves. Comparing the afterglow of GRB 111209A to this large sample can aid us in the determination of the progenitor. Again, we add published data to the light curve (Nysewander et al. 2011; Stratta et al. 2013, L14). At early times, as we have no overlapping observations, we assume that the $R_{C}$ data from TAROT are identical to our $r^{\prime}$ data (once they have been transformed to Vega magnitudes), then we shift the UVOT $v /$ white data points (these two agree very well with each other intrinsically) to the $R_{C}$ magnitudes, and adjust the rest of the UVOT data to this backbone. We also use UVOT data to fill the gap between the first and second day of GROND observations. At late times, we use the host-subtracted $U$ data for the post-break, SN-less afterglow. With a few exceptions, the resulting light curve shows little scatter. Furthermore, we shift the GROND $r^{\prime}$ magnitudes by 0.09-0.11 magnitudes (brighter, depending on the spectral slope) to transform them to $R_{C}$.

We add GRB 130925A as a direct comparison. The lightcurve treatment is detailed in Sect. 3.4.

In Fig. 10, we show the light curves of GRBs 111209A and 130925A, corrected for Galactic extinction and hostgalaxy contribution, but otherwise as observed, in comparison to the Kann et al. $(2010,2011 b)$ sample. The extreme variability during the prompt emission is evident in both GRBs. For GRB 111209A, starting with the peak of the flare at $\approx 2500$ s (Gendre et al. 2013; Stratta et al. 2013) which roughly corresponds to the brightest peak of the prompt emission (Golenetskii et al. 2011), the afterglow is at multiple times, and especially after the strong rebrightening we detected with GROND, among the brightest afterglows observed so far (see also Hoversten \& Siegel 2011). GRB 130925A, on the other hand, is extremely faint due to the very high line-of-sight extinction in the host galaxy (Greiner et al. 2014).

Similar to GRB 130925A (Sect. 3.4), we shift the optical light curve of GRB 111209A to $z=1$. Here, we are not interested in a detailed epoch-by-epoch shifting (which would probably simply increase the scatter), therefore we use only two spec- 


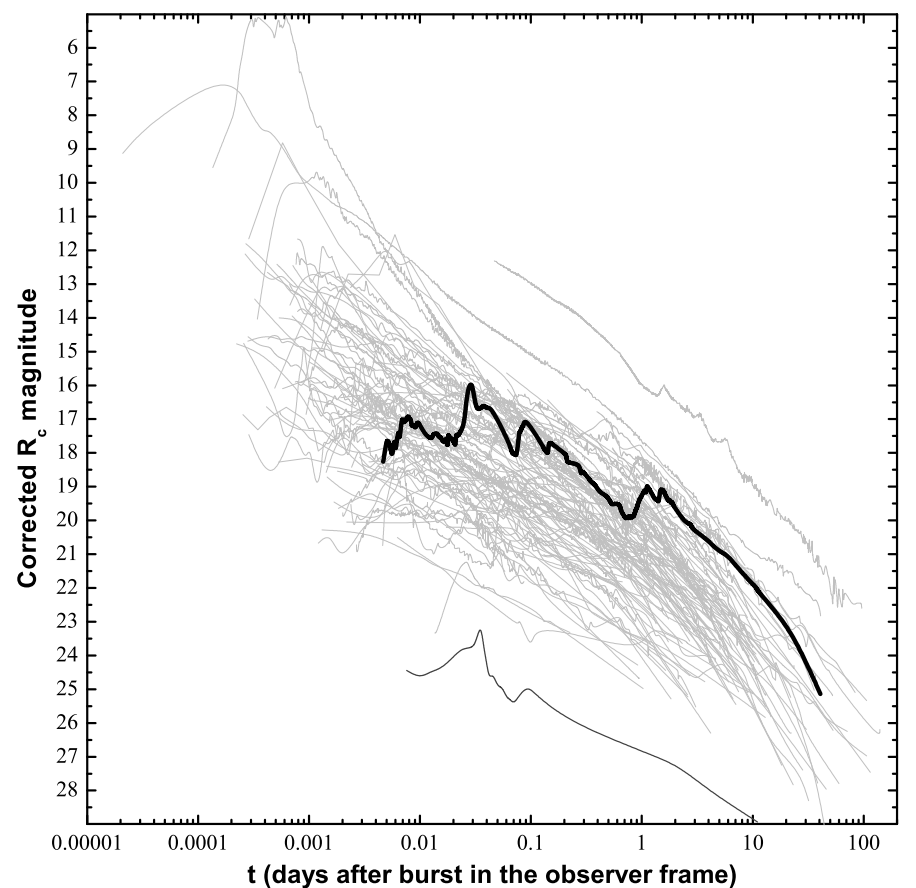

Fig. 10. $R$-band light curve of the afterglow of GRB 111209A in comparison to the long-GRB light-curve sample of Kann et al. (2010, 2011b). Data are corrected for Galactic extinction and, if possible, host galaxy contribution, but are otherwise as observed. The strong variability of the afterglow at early times is evident. At late times, the afterglow is among the brightest observed so far. This is in strong contrast to the $R$-band afterglow of GRB 130925A (thin black curve), which is exceedingly faint due to very high host-galaxy extinction (Greiner et al. 2014)

tral slopes to shift different parts of the light curve. We use the fit from the early prompt emission $(\beta=0.63 \pm 0.25$, $\left.A_{\mathrm{V}}=0.25 \pm 0.11\right)$ to shift data from the first two Swift orbits (up to 0.09 days), and the joint UVOT+GROND result $\left(\beta=1.05 \pm 0.06, A_{\mathrm{V}}=0.12 \pm 0.04\right)$ for the rest of the data. For the two parts, we find $d R c=+0.61_{-0.19}^{+0.20}$, and $d R c=+0.89_{-0.07}^{+0.06}$, respectively.

After this shift, we find (Fig. 11) that the afterglow of GRB 111209A lies well within the distribution of known afterglow magnitudes. Except for early times, when it actually belongs to the least luminous known afterglows, its luminosity is unremarkable (we find $R_{C}=20.75 \pm 0.07, M_{B}=-22.17 \pm 0.07$, $R_{C}=21.30 \pm 0.08, M_{B}=-21.62 \pm 0.08$ at one and four days after the trigger in the $z=1$ frame, respectively). Kann et al. (2010) compared a large number of long GRBs in terms of afterglow luminosity versus isotropic energy release (see also Gehrels et al. 2008; Nysewander et al. 2009), and the values for this GRB are not exceptional in this sense either (just as Golenetskii et al. 2011 have pointed out that the prompt emission is not really remarkable save for the duration). From an afterglow perspective, therefore, GRB 111209A is a typical long GRB, the only outstanding element being the strong variability, which is mostly linked to the extremely long prompt emission duration anyway.

The light curve of GRB 130925A is seen to resemble that of GRB 111209A quite remarkably in terms of temporal evolution. However, the actual luminosity of the afterglow is hard to determine (see Sect. 3.4 for more details), but the afterglow is likely to be less luminous than that of GRB 111209A, possibly significantly less so (as also deduced by Evans et al. 2014 upon comparison of the X-ray afterglows after correcting for the

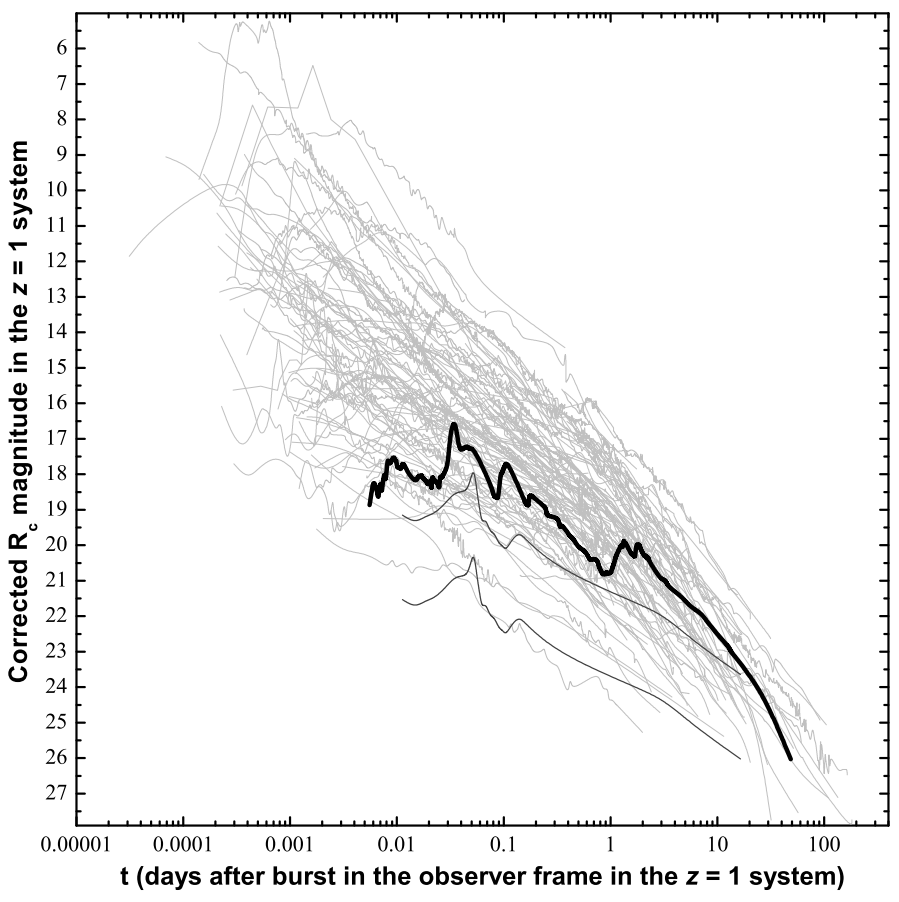

Fig. 11. As Fig. 10, but now in the $z=1$ system, where all light curves are directly comparable, having been corrected for all line-of-sight extinction and shifted to the same frame both in magnitude and time. The light curve of the afterglow of GRB 111209A is now seen to lie in the middle of the distribution of known afterglows, actually being among the least luminous at early times, and otherwise unremarkable from a luminosity standpoint. The afterglow of GRB 130925A is even fainter (the two different curves are based on two different extinction corrections; see text), and shows a remarkably similar evolution at early times.

dominating dust-echo component). The combination of high extinction and a bright host galaxy led to only sparse detections at late times; specifically, no attempt was made to detect the (likely) accompanying SN.

\section{Conclusions}

In this work, we have presented the detailed observations, obtained by GROND and UVOT, of the afterglow of the ultra-long duration GRB 111209A. Our main conclusions are the following: - Except for its extreme duration and slow variability, GRB 111209A is in agreement with usual GRBs. The prompt emission parameters are standard, and it agrees with the Amati relation (Amati et al. 2002), and with the Ghirlanda relation (Ghirlanda et al. 2004) assuming a low circumburst medium density, though there are indications this might not be the case (GF17).

- The afterglow shows a complicated evolution, featuring strong variability during the extremely long prompt emission phase. After a standard decay, a strong chromatic rebrightening follows, which we model with a two-component jet. The late afterglow also shows several smaller, achromatic rebrightenings, which are likely to be energy injections.

- In general, though, the afterglow of GRB 111209A is unremarkable in comparison to other GRB afterglows. This is in contrast to the long-wavelength transients following other ultra-long duration events, which were weaker or were not in agreement with synchrotron emission.

- In contrast to earlier work on this event, our dataset also reveals that this ultra-long duration GRB is accompanied by 
a very luminous supernova, which is spectrally dissimilar compared to usual GRB-SNe (G15,K18A). This SN is likely to be powered by a magnetar, and resembles SLSNe more than typical GRB-associated SNe. We find that, although very energetic, the entire event is still in agreement with being powered by a magnetar central engine.

- Our data puts to rest a lot of speculation on the nature of this special event, but a completely unified model explaining both the duration as well as the $\mathrm{SN}$ characteristics remains out of reach for now.

In multiple ways, GRB 111209A is an extraordinary event. Here, we have shown that in terms of the afterglow, it is not completely ordinary, but is clearly linked to standard GRBs and their emission mechanisms. Further dense multi-wavelength followup studies of this very rare class of GRBs will yield additional insight into the breadth of high-energy gamma-ray transients and their possible link to the most luminous stellar explosions.

Acknowledgements. DAK wishes to dedicate this work to his father, R.I.P. 20 08. 2015. You are sorely missed by so many. We thank the referee for the very valuable report which helped improve the clarity of the paper. DAK acknowledges Massimiliano De Pasquale, Daniele Malesani, Antonio de Ugarte Postigo, Christina C. Thöne, Thomas Kampf, Cristiano Guidorzi, and Raffaella Margutti for interesting discussions and helpful comments. DAK acknowledges financial support by the DFG Cluster of Excellence "Origin and Structure of the Universe," from MPE, from TLS, from the Spanish research project AYA 2014-58381-P, and from Juan de la Cierva Incorporación fellowship IJCI2015-26153. We are indebted to Joe Lyman and Vicki Toy for supplying the bolometric light curves of GRB 120422A/SN 2012bz and GRB 130702A/SN 2013dx, respectively. SK, DAK, ARossi, and ANG acknowledge support by DFG grants K1 766/16-1 and K1 766/16-3, SSchmidl also acknowledges the latter. ARossi acknowledges support from the Jenaer Graduiertenakademie and by the project PRIN-INAF 2012 "The role of dust in galaxy evolution". TK acknowledges support by the DFG Cluster of Excellence Origin and Structure of the Universe, and by the European Commission under the Marie Curie Intra-European Fellowship Programme. RF acknowledges support from European Regional Development Fund-Project "Engineering applications of microworld physics" (No. CZ.02.1.010.00.016_0190000766). DARK is funded by the DNRF. FOE acknowledges funding of his Ph.D. through the DAAD, and support from FONDECYT through postdoctoral grant 3140326. SSchulze acknowledges support from CONICYT-Chile FONDECYT 3140534, Basal-CATA PFB06/2007, and Project IC120009 "Millennium Institute of Astrophysics (MAS)" of Iniciativa Científica Milenio del Ministerio de Economía, Fomento y Turismo. SK, SSchmidl, and ANG acknowledge support by the Thüringer Ministerium für Bildung, Wissenschaft und Kultur under FKZ 12010-514. MN and PS acknowledge support by DFG grant SA 2001/2-1. ANG, DAK, ARossi and AU are grateful for travel funding support through MPE. Part of the funding for GROND (both hardware as well as personnel) was generously granted from the LeibnizPrize to Prof. G. Hasinger (DFG grant HA 1850/28-1). This work made use of data supplied by the UK Swift Science Data Centre at the University of Leicester.

\section{References}

Aguilera-Dena, D. R., Langer, N., Moriya, T. J., \& Schootemeijer, A. 2018, ApJ, 858,115

Aihara, H., Allende Prieto, C., An, D., et al. 2011, ApJS, 193, 29

Akerlof, C., Balsano, R., Barthelmy, S., et al. 1999, Nature, 398, 400

Alexander, K. D., Laskar, T., Berger, E., et al. 2017, ApJ, 848, 69

Amati, L., Frontera, F., Tavani, M., et al. 2002, A\&A, 390, 81

Arnouts, S., Cristiani, S., Moscardini, L., et al. 1999, MNRAS, 310, 540

Barthelmy, S. D., Barbier, L. M., Cummings, J. R., et al. 2005a, Space Sci. Rev., 120,143

Barthelmy, S. D., Cannizzo, J. K., Gehrels, N., et al. 2005b, ApJ, 635, L133

Basak, R., \& Rao, A. R. 2015, ApJ, 807, 34

Bellm, E. C., Barrière, N. M., Bhalerao, V., et al. 2014, ApJ, 784, L19

Beniamini, P., Nava, L., Duran, R. B., \& Piran, T. 2015, MNRAS, 454, 1073

Berger, E., Kulkarni, S. R., Pooley, G., et al. 2003, Nature, 426, 154

Beskin, G., Karpov, S., Bondar, S., et al. 2010, ApJ, 719, L10

Beuermann, K., Hessman, F. V., Reinsch, K., et al. 1999, A\&A, 352, L26

Björnsson, G., Gudmundsson, E. H., \& Jóhannesson, G. 2004, ApJ, 615, L77

Blake, C. H., Bloom, J. S., Starr, D. L., et al. 2005, Nature, 435, 181

Bloom, J. S., Kulkarni, S. R., \& Djorgovski, S. G. 2002, AJ, 123, 1111

Bloom, J. S., Perley, D. A., Li, W., et al. 2009, ApJ, 691, 723
Bloom, J. S., Giannios, D., Metzger, B. D., et al. 2011, Science, 333, 203 Boër, M., Gendre, B., \& Stratta, G. 2015a, ApJ, 800, 16

Boër, M., Gendre, B., \& Stratta, G. 2015b, Proc. "Swift 10 years of discovery" Conf., submitted, [arXiv:1504.03478]

Bromberg, O., Nakar, E., Piran, T., \& Sari, R. 2012, ApJ, 749, 110

Bromberg, O., Nakar, E., Piran, T., \& Sari, R. 2013, ApJ, 764, 179

Brown, G. C., Levan, A. J., Stanway, E. R., et al. 2015, MNRAS, 452, 4297

Brown, G. C., Levan, A. J., Stanway, E. R., et al. 2017, MNRAS, 472, 4469

Bufano, F., Pian, E., Sollerman, J., et al. 2012, ApJ, 753, 67

Burlon, D., Ghirlanda, G., Ghisellini, G., et al. 2008, ApJ, 685, L19

Burrows, D. N., Hill, J. E., Nousek, J. A., et al. 2005, Space Sci. Rev., 120, 165

Burrows, D. N., Kennea, J. A., Ghisellini, G., et al. 2011, Nature, 476, 421

Burrows, D. N., Malesani, D., Lien, A. Y., Cenko, S. B., \& Gehrels, N. 2013, GCN Circulars, 15253

Calzetti, D., Armus, L., Bohlin, R. C., et al. 2000, ApJ, 533, 682

Campana, S., Mangano, V., Blustin, A. J., et al. 2006, Nature, 442, 1008

Campana, S., Lodato, G., D'Avanzo, P., et al. 2011, Nature, 480, 69

Cano, Z., Bersier, D., Guidorzi, C., et al. 2011, ApJ, 740, 41

Cano, Z., Wang, S.-Q., Dai, Z.-G., \& Wu, X.-F. 2017, Adv. Astron., 2017, 8929054

Cardelli, J. A., Clayton, G. C., \& Mathis, J. S. 1989, ApJ, 345, 245

Castro-Tirado, A. J., Gorosabel, J., Benitez, N., et al. 1998, Science, 279, 1011

Cenko, S. B., Kasliwal, M., Harrison, F. A., et al. 2006, ApJ, 652, 490

Cenko, S. B., Frail, D. A., Harrison, F. A., et al. 2011, ApJ, 732, 29

Cenko, S. B., Krimm, H. A., Horesh, A., et al. 2012, ApJ, 753, 77

Chandra, P., Cenko, S. B., Frail, D. A., et al. 2008, ApJ, 683, 924

Chornock, R., Berger, E., Levesque, E. M., et al. 2010, ArXiv e-prints [arXiv: 1004.2262]

Cobb, B. E., Bailyn, C. D., van Dokkum, P. G., \& Natarajan, P. 2006, ApJ, 645, L113

Connaughton, V., Kippen, R. M., Preece, R., \& Hurley, K. 1997, IAU Circ., 6785

Costa, E., Frontera, F., Heise, J., et al. 1997, Nature, 387, 783

Cucchiara, A., Veres, P., Corsi, A., et al. 2015, ApJ, 812, 122

D'Ai, A., Burrows, D. N., Cholden-Brown, A., et al. 2017, GRB Coordinates Network, Circular Service, 21340

Dai, Z. G., \& Lu, T. 1998, A\&A, 333, L87

D’Avanzo, P., D’Elia, V., Cholden-Brown, A., et al. 2017, GRB Coordinates Network, Circular Service, 21343

De Pasquale, M., Page, M. J., Kann, D. A., et al. 2016, MNRAS, 462, 1111

de Ugarte Postigo, A., Castro-Tirado, A. J., Gorosabel, J., et al. 2005, A\&A, 443, 841

de Ugarte Postigo, A., Kann, D. A., Izzo, L., et al. 2017a, GRB Coordinates Network, Circular Service, 21346

de Ugarte Postigo, A., Kann, D. A., Izzo, L., et al. 2017b, GRB Coordinates Network, Circular Service, 21359

de Ugarte Postigo, A., Kann, D. A., Schulze, S., et al. 2017c, GRB Coordinates Network, Circular Service, 21356

Djorgovski, S. G., Metzger, M. R., Kulkarni, S. R., et al. 1997, Nature, 387, 876 Elíasdóttir, Á., Fynbo, J. P. U., Hjorth, J., et al. 2009, ApJ, 697, 1725

Elliott, J., Yu, H.-F., Schmidl, S., et al. 2014, A\&A, 562, A100

Evans, P. A., Beardmore, A. P., Page, K. L., et al. 2007, A\&A, 469, 379

Evans, P. A., Beardmore, A. P., Page, K. L., et al. 2009, MNRAS, 397, 1177

Evans, P. A., Page, K. L., \& Osborne, J. P. 2012, GCN Circulars, 13921

Evans, P. A., Pagani, C., Page, K. L., et al. 2013, GCN Circulars, 15254

Evans, P. A., Willingale, R., Osborne, J. P., et al. 2014, MNRAS, 444, 250

Fenimore, E., Barthelmy, S., Cummings, J., et al. 2004, GCN Circulars, 2906

Ferrero, P., Kann, D. A., Zeh, A., et al. 2006, A\&A, 457, 857

Ferrero, P., Klose, S., Kann, D. A., et al. 2009, A\&A, 497, 729

Filgas, R., Greiner, J., Schady, P., et al. 2011a, A\&A, 535, A57

Filgas, R., Krühler, T., Greiner, J., et al. 2011b, A\&A, 526, A113

Filgas, R., Greiner, J., Schady, P., et al. 2012, A\&A, 546, A101

Fitzpatrick, G. 2013, GCN Circulars, 15255

Frail, D. A., Kulkarni, S. R., Nicastro, L., Feroci, M., \& Taylor, G. B. 1997, Nature, 389, 261

Friedman, A. S., \& Bloom, J. S. 2005, ApJ, 627, 1

Fugazza, D., Covino, S., Palazzi, E., \& Antonelli, L. A. 2011, GCN Circulars, 12634

Fynbo, J. U., Gorosabel, J., Dall, T. H., et al. 2001, A\&A, 373, 796

Fynbo, J. P. U., Jakobsson, P., Prochaska, J. X., et al. 2009, ApJS, 185, 526

Gao, H., \& Mészáros, P. 2015, ApJ, 802, 90

Gao, H., Lei, W.-H., Zou, Y.-C., Wu, X.-F., \& Zhang, B. 2013, New Astron. Rev., 57, 141

Gao, H., Ren, A.-B., Lei, W.-H., et al. 2017, ApJ, 845, 51

Gehrels, N., Chincarini, G., Giommi, P., et al. 2004, ApJ, 611, 1005

Gehrels, N., Barthelmy, S. D., Burrows, D. N., et al. 2008, ApJ, 689, 1161

Gendre, B., Stratta, G., Atteia, J. L., et al. 2013, ApJ, 766, 30

Ghirlanda, G., Ghisellini, G., \& Lazzati, D. 2004, ApJ, 616, 331 
Ghirlanda, G., Ghisellini, G., Firmani, C., et al. 2006, A\&A, 452, 839 Ghisellini, G., Ghirlanda, G., Nava, L., \& Firmani, C. 2007, ApJ, 658, L75 Golenetskii, S., Aptekar, R., Mazets, E., et al. 2011, GCN Circulars, 12663 Golenetskii, S., Aptekar, R., Frederiks, D., et al. 2013, GCN Circulars, 15260 Golenetskii, S., Aptekar, R., Frederiks, D., et al. 2014, GRB Coordinates Network, 17108

Gomboc, A., Kobayashi, S., Guidorzi, C., et al. 2008, ApJ, 687, 443

Gompertz, B., \& Fruchter, A. 2017, ApJ, 839, 49

Granot, J., Nakar, E., \& Piran, T. 2003, Nature, 426, 138

Granot, J., Königl, A., \& Piran, T. 2006, MNRAS, 370, 1946

Greiner, J., Bornemann, W., Clemens, C., et al. 2008, PASP, 120, 405

Greiner, J., Krühler, T., McBreen, S., et al. 2009, ApJ, 693, 1912

Greiner, J., Krühler, T., Nardini, M., et al. 2013, A\&A, 560, A70

Greiner, J., Yu, H.-F., Krühler, T., et al. 2014, A\&A, 568, A75

Greiner, J., Mazzali, P. A., Kann, D. A., et al. 2015, Nature, 523, 189

Gruber, D., Krühler, T., Foley, S., et al. 2011, A\&A, 528, A15

Grupe, D., \& Hoversten, E. A. 2011, GCN Circulars, 12643

Guidorzi, C., Kobayashi, S., Perley, D. A., et al. 2011a, MNRAS, 417, 2124

Guidorzi, C., Melandri, A., Kobayashi, S., Mundell, C. G., \& Gomboc, A. 2011b, GCN Circulars, 12635

Hamidani, H., Takahashi, K., Umeda, H., \& Okita, S. 2017, MNRAS, 469, 2361

Hancock, P. J., Murphy, T., Gaensler, B., \& Zauderer, A. 2011, GCN Circulars, 12664

Hancock, P. J., Murphy, T., Gaensler, B., \& Zauderer, A. 2012, GCN Circulars, 12804

Hjorth, J., \& Bloom, J. S. 2012, The Gamma-Ray Burst - Supernova Connection (Cambridge: Cambridge University Press), 169

Holland, S. T., Sbarufatti, B., Shen, R., et al. 2010, ApJ, 717, 223

Horesh, A., Cenko, S. B., Perley, D. A., et al. 2015, ApJ, 812, 86

Horesh, A., Cenko, S. B., Levan, A., \& Tanvir, N. 2017, GRB Coordinates Network, Circular Service, 21352

Hou, S.-J., Gao, H., Liu, T., et al. 2014, MNRAS, 441, 2375

Hou, S.-J., Liu, T., Xu, R.-X., et al. 2018, ApJ, 854, 104

Hoversten, E. A., \& Siegel, M. H. 2011, GCN Circulars, 12642

Hoversten, E. A., Burrows, D. N., Campana, S., et al. 2011a, GCN Circulars, 12641

Hoversten, E. A., Evans, P. A., Guidorzi, C., et al. 2011b, GCN Circulars, 12632

Huang, Y. F., Cheng, K. S., \& Gao, T. T. 2006, ApJ, 637, 873

Hurley, K., Golenetskii, S., Aptekar, R., et al. 2013, GCN Circulars, 15278

Ilbert, O., Arnouts, S., McCracken, H. J., et al. 2006, A\&A, 457, 841

Im, M., Jun, H., \& Kim, D. 2011, GCN Circulars, 12649

Inserra, C., Nicholl, M., Chen, T.-W., et al. 2017, MNRAS, 468, 4642

Jakobsson, P., Hjorth, J., Malesani, D., et al. 2012, ApJ, 752, 62

Jones, D. H., Read, M. A., Saunders, W., et al. 2009, MNRAS, 399, 683

Kann, D. A., \& Greiner, J. 2011, GCN Circulars, 12656

Kann, D. A., Klose, S., \& Zeh, A. 2006, ApJ, 641, 993

Kann, D. A., Masetti, N., \& Klose, S. 2007, AJ, 133, 1187

Kann, D. A., Klose, S., Zhang, B., et al. 2010, ApJ, 720, 1513

Kann, D. A., Klose, S., Krühler, T., \& Greiner, J. 2011a, GCN Circulars, 12647

Kann, D. A., Klose, S., Zhang, B., et al. 2011b, ApJ, 734, 96

Kann, D. A., Schady, P., Olivares E., F., et al. 2016, A\&A, submitted, [arXiv:1606.06791v3]

Kann, D. A., de Ugarte Postigo, A., Izzo, L., \& Thoene, C. C. 2017, GRB Coordinates Network, Circular Service, 21345

Kashiyama, K., Nakauchi, D., Suwa, Y., Yajima, H., \& Nakamura, T. 2013, ApJ, 770,8

Klebesadel, R. W., Strong, I. B., \& Olson, R. A. 1973, ApJ, 182, L85

Klotz, A., Gendre, B., Boër, M., \& Atteia, J. L. 2011a, GCN Circulars, 12633

Klotz, A., Gendre, B., Boër, M., \& Atteia, J. L. 2011b, GCN Circulars, 12637

Kouveliotou, C., Meegan, C. A., Fishman, G. J., et al. 1993, ApJ, 413, L101

Krühler, T., Küpcü Yoldaş, A., Greiner, J., et al. 2008, ApJ, 685, 376

Krühler, T., Greiner, J., McBreen, S., et al. 2009, ApJ, 697, 758

Krühler, T., Malesani, D., Fynbo, J. P. U., et al. 2015, A\&A, 581, A125

Kumar, P., \& Panaitescu, A. 2000, ApJ, 541, L51

Kumar, P., \& Piran, T. 2000, ApJ, 532, 286

Kumar, P., \& Barniol Duran, R. 2010, MNRAS, 409, 226

Laskar, T., Berger, E., Zauderer, B. A., et al. 2013, ApJ, 776, 119

Laskar, T., Berger, E., Margutti, R., et al. 2015, ApJ, 814, 1

Laskar, T., Alexander, K. D., Berger, E., et al. 2016, ApJ, 833, 88

Lazzati, D., Villeneuve, M., López-Cámara, D., Morsony, B. J., \& Perna, R. 2013, MNRAS, 436, 1867

Levan, A. J. 2015, J. High Energy Astrophys., 7, 44

Levan, A. J., Tanvir, N. R., Cenko, S. B., et al. 2011, Science, 333, 199

Levan, A. J., Read, A. M., Metzger, B. D., Wheatley, P. J., \& Tanvir, N. R. 2013, ApJ, 771, 136

Levan, A. J., Tanvir, N. R., Starling, R. L. C., et al. 2014, ApJ, 781, 13

Liang, E. W., Zhang, B., O'Brien, P. T., et al. 2006, ApJ, 646, 351
Lien, A., Sakamoto, T., Barthelmy, S. D., et al. 2016, ApJ, 829, 7

Lin, D.-B., Huang, B.-Q., Liu, T., et al. 2018, ApJ, 852, 136

Lipkin, Y. M., Ofek, E. O., Gal-Yam, A., et al. 2004, ApJ, 606, 381

Lü, H.-J., Lan, L., Zhang, B., et al. 2018, ApJ, 862, 130

MacFadyen, A. I., \& Woosley, S. E. 1999, ApJ, 524, 262

Margalit, B., \& Metzger, B. D. 2017, ApJ, 850, L19

Margutti, R., Soderberg, A. M., Wieringa, M. H., et al. 2013, ApJ, 778, 18

Margutti, R., Guidorzi, C., Lazzati, D., et al. 2015, ApJ, 805, 159

Margutti, R., Chornock, R., Metzger, B. D., et al. 2017, ApJ, 864, 45

Markwardt, C. B., Barthelmy, S. D., Baumgartner, W. H., et al. 2013, GCN Circulars, 15257

Maselli, A., Melandri, A., Nava, L., et al. 2014, Science, 343, 48

Maza, J., Ruiz, M. T., Gonzalez, L. E., Wischnjewsky, M., \& Antezana, R. 1993, Rev. Mex. Astron. Astrofis., 25, 51

Mazets, E. P., Golenetskii, S. V., Ilinskii, V. N., et al. 1981, Ap\&SS, 80, 3

Mazzali, P. A., McFadyen, A. I., Woosley, S. E., Pian, E., \& Tanaka, M. 2014, MNRAS, 443, 67

Mazzali, P. A., Sullivan, M., Pian, E., Greiner, J., \& Kann, D. A. 2016, MNRAS, 458, 3455

Metzger, M. R., Djorgovski, S. G., Kulkarni, S. R., et al. 1997, Nature, 387, 878

Metzger, B. D., Margalit, B., Kasen, D., \& Quataert, E. 2015, MNRAS, 454, 3311

Metzger, B. D., Beniamini, P., \& Giannios, D. 2018, ApJ, 857, 95

Mirabal, N., Halpern, J. P., An, D., Thorstensen, J. R., \& Terndrup, D. M. 2006, ApJ, 643, L99

Modjaz, M., Stanek, K. Z., Garnavich, P. M., et al. 2006, ApJ, 645, L21

Molinari, E., Vergani, S. D., Malesani, D., et al. 2007, A\&A, 469, L13

Monfardini, A., Kobayashi, S., Guidorzi, C., et al. 2006, ApJ, 648, 1125

Morgan, A. N., Perley, D. A., Cenko, S. B., et al. 2014, MNRAS, 440, 1810

Nakauchi, D., Kashiyama, K., Suwa, Y., \& Nakamura, T. 2013, ApJ, 778, 67

Nardini, M., Greiner, J., Krühler, T., et al. 2011, A\&A, 531, A39

Nardini, M., Elliott, J., Filgas, R., et al. 2014, A\&A, 562, A29

Nava, L., Ghisellini, G., Ghirlanda, G., Tavecchio, F., \& Firmani, C. 2006, A\&A, 450,471

Nicastro, L., in't Zand, J. J. M., Amati, L., et al. 2004, A\&A, 427, 445

Nicholl, M., Guillochon, J., \& Berger, E. 2017, ApJ, 850, 55

Nysewander, M., Fruchter, A. S., \& Pe'er, A. 2009, ApJ, 701, 824

Nysewander, M., Haislip, J., Lacluyze, A., et al. 2011, GCN Circulars, 12645

Oates, S. R., Page, M. J., Schady, P., et al. 2009, MNRAS, 395, 490

Olivares E., F., Greiner, J., Schady, P., et al. 2012, A\&A, 539, A76

Page, K. L., Willingale, R., Osborne, J. P., et al. 2007, ApJ, 663, 1125

Palmer, D. M., Barthelmy, S. D., Baumgartner, W. H., et al. 2011, GCN Circulars, 12640

Palmer, D. M., Barthelmy, S. D., Cummings, J. R., et al. 2017, GRB Coordinates Network, Circular Service, 21347

Pal'Shin, V., Aptekar, R., Frederiks, D., et al. 2008, AIP Conf. Ser., 1000, 117

Pal'shin, V., Hurley, K., Goldsten, J., et al. 2012, in Gamma-Ray Bursts 2012 Conf. (GRB 2012), 40

Panaitescu, A., Meszaros, P., \& Rees, M. J. 1998, ApJ, 503, 314

Panaitescu, A., Vestrand, W. T., \& Woźniak, P. 2013, MNRAS, 436, 3106

Pasham, D. R., Cenko, S. B., Levan, A. J., et al. 2015, ApJ, 805, 68

Pei, Y. C. 1992, ApJ, 395, 130

Peng, F., Königl, A., \& Granot, J. 2005, ApJ, 626, 966

Peng, F.-K., Hu, Y.-D., Xi, S.-Q., et al. 2013, ArXiv e-prints [arXiv: 1302 .4876]

Perley, D. A., Bloom, J. S., Butler, N. R., et al. 2008a, ApJ, 672, 449

Perley, D. A., Li, W., Chornock, R., et al. 2008b, ApJ, 688, 470

Perley, D. A., Morgan, A. N., Updike, A., et al. 2011, AJ, 141, 36

Perley, D. A., Cenko, S. B., Corsi, A., et al. 2014, ApJ, 781, 37

Perna, R., Lazzati, D., \& Cantiello, M. 2018, ApJ, 859, 48

Pian, E., Mazzali, P. A., Masetti, N., et al. 2006, Nature, 442, 1011

Piro, L., Troja, E., Gendre, B., et al. 2014, ApJ, 790, L15

Piro, L., Ricci, R., Wieringa, M., et al. 2017, GRB Coordinates Network, Circular Service, 21424

Poole, T. S., Breeveld, A. A., Page, M. J., et al. 2008, MNRAS, 383, 627

Prajs, S., Sullivan, M., Smith, M., et al. 2017, MNRAS, 464, 3568

Racusin, J. L., Karpov, S. V., Sokolowski, M., et al. 2008, Nature, 455, 183

Rau, A., Savaglio, S., Krühler, T., et al. 2010, ApJ, 720, 862

Rees, M. J., \& Meszaros, P. 1998, ApJ, 496, L1

Rees, M. J., \& Mészáros, P. 2000, ApJ, 545, L73

Rezzolla, L., Most, E. R., \& Weih, L. R. 2018, ApJ, 852, L25

Rhoads, J. E. 1997, ApJ, 487, L1

Rhoads, J. E. 1999, ApJ, 525, 737

Ricci, R., Wieringa, M., Piro, L., et al. 2017, GRB Coordinates Network, Circular Service, 21360

Romano, P., Campana, S., Chincarini, G., et al. 2006, A\&A, 456, 917

Roming, P. W. A., Kennedy, T. E., Mason, K. O., et al. 2005, Space Sci. Rev., 120,95 
Rossi, A., Schulze, S., Klose, S., et al. 2011, A\&A, 529, A142 Ruiz, M., Shapiro, S. L., \& Tsokaros, A. 2018, Phys. Rev. D, 97, 021501 Ryan, G., van Eerten, H., MacFadyen, A., \& Zhang, B.-B. 2015, ApJ, 799, 3 Santana, R., Barniol Duran, R., \& Kumar, P. 2014, ApJ, 785, 29

Sari, R., \& Mészáros, P. 2000, ApJ, 535, L33

Sari, R., Piran, T., \& Narayan, R. 1998, ApJ, 497, L17

Sari, R., Piran, T., \& Halpern, J. P. 1999, ApJ, 519, L17

Savchenko, V., Beckmann, V., Ferrigno, C., et al. 2013, GCN Circulars, 15259

Schady, P., Krühler, T., Greiner, J., et al. 2015, A\&A, 579, A126

Schlegel, D. J., Finkbeiner, D. P., \& Davis, M. 1998, ApJ, 500, 525

Schulze, S., Malesani, D., Cucchiara, A., et al. 2014, A\&A, 566, A102

Serino, M., Nakahira, S., Negoro, H., et al. 2012, GCN Circulars, 13908

Shen, R., Kumar, P., \& Robinson, E. L. 2006, MNRAS, 371, 1441

Shibata, M., Fujibayashi, S., Hotokezaka, K., et al. 2017, Phys. Rev. D, 96 , 123012

Siegel, M. H., Evans, P. A., Oates, S. R., N. Burrows, S. D., \& Gehrels, N. 2013, GCN Report, 410

Skrutskie, M. F., Cutri, R. M., Stiening, R., et al. 2006, AJ, 131, 1163

Sokolowski, M., Majcher, A., Batsch, T., et al. 2011, GCN Circulars, 12646

Sollerman, J., Jaunsen, A. O., Fynbo, J. P. U., et al. 2006, A\&A, 454, 503

Spergel, D. N., Verde, L., Peiris, H. V., et al. 2003, ApJS, 148, 175

Starling, R. L. C. 2008, A\&A, 488, 915

Starling, R. L. C., O'Brien, P. T., Willingale, R., et al. 2008, MNRAS, 384, 504

Starling, R. L. C., Wiersema, K., Levan, A. J., et al. 2011, MNRAS, 411, 2792

Starling, R. L. C., Page, K. L., Pe'er, A., Beardmore, A. P., \& Osborne, J. P. 2012, MNRAS, 427, 2950

Stratta, G., Gendre, B., Atteia, J. L., et al. 2013, ApJ, 779, 66

Sudilovsky, V., Kann, D. A., \& Greiner, J. 2013a, GCN Circulars, 15247

Sudilovsky, V., Kann, D. A., Schady, P., et al. 2013b, GCN Circulars, 15250

Suzuki, K., Sakakibara, H., Negoro, H., et al. 2013, GCN Circulars, 15248

Tagliaferri, G., Goad, M., Chincarini, G., et al. 2005, Nature, 436, 985

Tanvir, N. R., Levan, A. J., Hounsell, R., et al. 2013, GCN Circulars, 15489

Thöne, C. C., Kann, D. A., Jóhannesson, G., et al. 2010, A\&A, 523, A70

Thöne, C. C., de Ugarte Postigo, A., Fryer, C. L., et al. 2011, Nature, 480, 72

Tikhomirova, Y. Y., \& Stern, B. E. 2005, Astron. Lett., 31, 291

Tody, D. 1993, ASP Conf. Ser., 52, 173

Troja, E., Lipunov, V. M., Mundell, C. G., et al. 2017, Nature, 547, 425

Uemura, M., Kato, T., Ishioka, R., et al. 2003, Nature, 423, 843

Updike, A. C., Haislip, J. B., Nysewander, M. C., et al. 2008, ApJ, 685, 361

van Paradijs, J., Groot, P. J., Galama, T., et al. 1997, Nature, 386, 686

Vernet, J., Dekker, H., D’Odorico, S., et al. 2011, A\&A, 536, A105

Vestrand, W. T., Wozniak, P. R., Wren, J. A., et al. 2005, Nature, 435, 178

Vestrand, W. T., Wren, J. A., Wozniak, P. R., et al. 2006, Nature, 442, 172

Vestrand, W. T., Wren, J. A., Panaitescu, A., et al. 2014, Science, 343, 38

Virgili, F. J., Mundell, C. G., Pal'shin, V., et al. 2013, ApJ, 778, 54

Vreeswijk, P., Fynbo, J., \& Melandri, A. 2011, GCN Circulars, 12648

Vreeswijk, P. M., Malesani, D., Fynbo, J. P. U., De Cia, A., \& Ledoux, C. 2013 GCN Circulars, 15249

Wang, S.-Q., Cano, Z., Wang, L.-J., et al. 2017, ApJ, 850, 148

Wang, X.-G., Zhang, B., Liang, E.-W., et al. 2015, ApJS, 219, 9

Wang, X.-G., Zhang, B., Liang, E.-W., et al. 2018, ApJ, 859, 160

Waxman, E., \& Draine, B. T. 2000, ApJ, 537, 796

Woosley, S. E. 1993, ApJ, 405, 273

Woosley, S. E., \& Bloom, J. S. 2006, ARA\&A, 44, 507

Woźniak, P. R., Vestrand, W. T., Wren, J. A., et al. 2006, ApJ, 642, L99

Woźniak, P. R., Vestrand, W. T., Panaitescu, A. D., et al. 2009, ApJ, 691, 495

Wren, J., Vestrand, W. T., Wozniak, P., \& Davis, H. 2011, GCN Circulars, 12684

Wu, X.-F., Hou, S.-J., \& Lei, W.-H. 2013, ApJ, 767, L36

Yoldaş, A. K., Krühler, T., Greiner, J., et al. 2008, in AIP Conf. Ser., 100, 227

Yu, Y. B., Wu, X. F., Huang, Y. F., et al. 2015, MNRAS, 446, 3642

Yu, Y.-W., \& Li, S.-Z. 2017, MNRAS, 470, 197

Yu, Y.-W., Zhu, J.-P., Li, S.-Z., Lü, H.-J., \& Zou, Y.-C. 2017, ApJ, 840, 12

Zafar, T., Watson, D., Elíasdóttir, Á., et al. 2012, ApJ, 753, 82

Zauderer, B. A., Berger, E., Soderberg, A. M., et al. 2011, Nature, 476 , 425

Zeh, A., Klose, S., \& Kann, D. A. 2006, ApJ, 637, 889
Zhang, B., \& Kumar, P. 2013, Phys. Rev. Lett., 110, 121101

Zhang, B., \& Mészáros, P. 2001, ApJ, 552, L35

Zhang, B., \& Mészáros, P. 2002, ApJ, 566, 712

Zhang, B., \& Mészáros, P. 2004, Int. J. Mod. Phys. A, 19, 2385

Zhang, B., Liang, E., Page, K. L., et al. 2007, ApJ, 655, 989

Zhang, B., Zhang, B.-B., Virgili, F. J., et al. 2009, ApJ, 703, 1696

Zhang, B.-B., Burrows, D. N., Zhang, B., et al. 2012, ApJ, 748, 132

Zhang, B.-B., Zhang, B., Murase, K., Connaughton, V., \& Briggs, M. S. 2014, ApJ, 787, 66

Zhang, B.-B., Zhang, B., Castro-Tirado, A. J., et al. 2018, Nat. Astron., 2, 69

Zhao, Y.-N., \& Shao, L. 2014, ApJ, 789, 74

Zou, Y. C., Dai, Z. G., \& Xu, D. 2006, ApJ, 646, 1098

1 Thüringer Landessternwarte Tautenburg, Sternwarte 5, 07778 Tautenburg, Germany e-mail: kann@iaa.es

2 Max-Planck-Institut für extraterrestrische Physik, Giessenbachstraße 1, 85748 Garching, Germany

3 Universe Cluster, Technische Universität München, Boltzmannstraße 2, 85748 Garching, Germany

4 Instituto de Astrofísica de Andalucía (IAA-CSIC), Glorieta de la Astronomía s/n, 18008 Granada, Spain

5 Departamento de Ciencias Físicas, Universidad Andres Bello, Avda. República 252, Santiago, Chile

6 INAF-IASF Bologna, Area della Ricerca CNR, via Gobetti 101, 40129 Bologna, Italy

7 Cahill Center for Astrophysics, California Institute of Technology, Pasadena, CA 91125, USA

8 Dark Cosmology Centre, Niels Bohr Institute, University of Copenhagen, Juliane Maries Vej 30, 2100 Copenhagen, Denmark

9 Astrophysics Research Institute, Liverpool John Moores University, IC2, Liverpool Science Park, 146 Brownlow Hill, Liverpool L3 5RF, UK

10 Department of Physics and Astronomy, University of Nevada Las Vegas, Las Vegas, NV 89154, USA

11 ESO, Alonso de Córdova 3107, Vitacura, Santiago de Chile, Chile

12 Harvard-Smithsonian Center for Astrophysics, 60 Garden St., Cambridge, MA 02138, USA

13 Institute of Experimental and Applied Physics, Czech Technical University in Prague, Horská 3a/22, 12800 Prague, Czech Republic

14 Scuola Normale Superiore, Piazza dei Cavalieri 7, 56126 Pisa, Italy

15 Astrophysics Research Institute, Liverpool John Moores University, 146 Brownlow Hill, Liverpool L3 5RF, UK

16 Max-Planck Institut für Astrophysik, Karl-Schwarzschild-Str. 1, 85748 Garching, Germany

17 Department of Particle Physics \& Astrophysics, Weizmann Institute of Science, Rehovot 76100, Israel

18 American River College, Physics and Astronomy Dpt., 4700 College Oak Drive, Sacramento, CA 95841, USA

19 Instituto de Astrofísica, Facultad de Física, Pontificia Universidad Católica de Chile, Av. Vicuña Mackenna 4860, 306, Santiago 22, Chile

20 Millennium Institute of Astrophysics, Av. Vicuña Mackenna 4860, 7820436 Macul, Santiago, Chile

21 Department of Particle Physics and Astrophysics, Faculty of Physics, Weizmann Institute of Science, Rehovot 76100, Israel

22 Department of Chemistry and Physics, Roger Williams University, One Old Ferry Road, Bristol, RI 02809, USA 


\section{Appendix A: Initial observations of GRB 111209A}

GRB 111209A was localized as Trigger \#509336 on 9 December 2011 at 07:12:08 (UT times are used throughout the paper) by the Swift satellite (Gehrels et al. 2004) with its high-energy Burst Alert Telescope (BAT, Barthelmy et al. 2005a), which slewed immediately to follow it up with its narrow-field instruments, the X-Ray Telescope (XRT, Burrows et al. 2005) and the UltraViolet-Optical Telescope (UVOT, Roming et al. 2005). The initial observation report already included the discovery of an X-ray and an optical/UV afterglow (Hoversten et al. 2011b). The initial detection by BAT was based on an image of 320 $\mathrm{s}$ exposure, indicating a long-lasting, low peak-flux event. At $T_{0}=+424 \mathrm{~s}$, Swift triggered a second time (\#509337), a $64 \mathrm{~s}$ image trigger at much higher flux. Such a double trigger was only known from GRB $110709 \mathrm{~B}$ beforehand ${ }^{12}$, according to Zhang et al. (2012). By this time, the satellite was already observing with its narrow-field instruments.

Rapid ground-based follow-up within minutes of the alert was reported from the TAROT South (Klotz et al. 2011a, see also Gendre et al. 2013; Stratta et al. 2013), REM (Fugazza et al. 2011, both stationed at La Silla, Chile), FTN (Guidorzi et al. 2011b, stationed on Hawaii) and PROMPT (Nysewander et al. 2011, stationed at Cerro Tololo, Chile) telescopes, whereas no bright flashes were seen in wide-field surveys (Sokolowski et al. 2011; Wren et al. 2011). Continued observations revealed the GRB remained bright and highly variable at gamma-ray (Palmer et al. 2011), X-ray (Grupe \& Hoversten 2011) and UV/optical wavelengths (Klotz et al. 2011b; Hoversten \& Siegel 2011), leading the Swift team to declare this as a "Burst of Interest" (Hoversten et al. 2011a). Spectroscopy by VLT X-shooter revealed the GRB to lie at $z=0.677$ (Vreeswijk et al. 2011, note that Im et al. 2011 also report NIR spectroscopy with IRTF SpeX but do not report any line detections), which is relatively close for a Swift GRB (Fynbo et al. 2009; Jakobsson et al. 2012), and simultaneously ruled out a peculiar Galactic transient (which was not suspected anyway due to the high Galactic latitude) as well as an event at very high redshift, in which case time dilation would have strongly contributed to the duration (the low redshift was also already suspected due to the detection in all UVOT filters, Hoversten \& Siegel 2011). Furthermore, after an initial non-detection (Hancock et al. 2011), a bright radio afterglow was also reported by Hancock et al. (2012).

The true dimension of GRB 111209A was finally revealed by the observations of the Konus detector on the WIND spacecraft (Golenetskii et al. 2011) in combination with the X-ray light curve ${ }^{13}$. The GRB is seen to begin $\approx 5400 \mathrm{~s}$ before the Swift trigger time ${ }^{14}$, and extend to $\approx 10000 \mathrm{~s}$. The XRT observations reveal that the X-ray emission remains roughly constant (with several flares superposed) up to $\approx 20000 \mathrm{~s}$, then a steep decay sets in. This is usually attributed to high-latitude emission and marks the point where the central engine "turns off", and thus the prompt emission ends (Kumar \& Panaitescu 2000; Tagliaferri et al. 2005; Barthelmy et al. 2005b; Zhang et al. 2014). Therefore, the prompt emission duration of the entire event is around $25000 \mathrm{~s}$, or seven hours (see also Gendre et al. 2013; Lien et al. 2016 detect the event for $\approx 18000$ s post-

\footnotetext{
12 The TDE GRB 110328A/Swift J164449.3+573451 also caused a double trigger but was not actually a GRB (Levan et al. 2011).

13 On the Swift XRT repository (Evans et al. 2007, 2009) webpage at http://www.swift.ac.uk/xrt_curves/00509336/

14 Additionally, there is a possible precursor at $\approx-10000 \mathrm{~s}$, but this burst has not been conclusively linked to GRB 111209A.
}

trigger in BAT survey data), significantly longer than even GRB 060814B (about four hours long, Pal'Shin et al. 2008, see Appendix B). Golenetskii et al. (2011) also reported that the other prompt emission parameters, such as the spectral shape and the isotropic energy release, were within the typical distribution of GRBs (see also our own analysis in Sect. 4.1). While the peak flux of the GRB is low, the extreme duration leads to a large total fluence; the comparably low redshift, though, implies a large but by no means exceptional total energy release. The Konus-WIND light curve ${ }^{15}$ indeed resembles that of a typical GRB, except that it is actually stretched by several orders of magnitude. Morphologically, it is therefore most similar to the first "class" mentioned in Appendix B (and "continuous" in the classification of Virgili et al. 2013).

\section{Appendix B: Examples of GRBs of extreme duration and a rough phenomenological classification scheme}

So far, there is no fixed definition for the labels "extremely long duration" (which we shorten to EL-GRBs) and "ultra-long" (the special class of ULGRBs, L14). Such a definition would be arbitrary anyway, especially since $T_{90}$ (defined as the time over which $90 \%$ of the fluence is accumulated, starting after the first 5\% and ending at 95\%) is an observer-frame quantity (and also strongly detector-dependent). Numbers which could be used are $600 \mathrm{~s}$ (i.e. $10 \mathrm{~min}$ ), and 1000 s. For actual "ultra-long" GRBs as presented by L14, we suggest a redshift-corrected duration of prompt emission activity (Zhang et al. 2014) of at least one hour.

- The most "generic" EL-GRBs consist of multiple spacedapart emission episodes of roughly similar intensity. Tikhomirova \& Stern (2005) have found multiple cases in the data of the Burst And Transient Source Experiment (BATSE) on the Compton Gamma-Ray Observatory. Further individual examples are GRB 020410 (Nicastro et al. 2004, $T_{90} \approx 1500 \mathrm{~s}$ ), the IPN GRB 080407 (Pal'shin et al. 2012, with $T_{90} \approx 2100 \mathrm{~s}$ ), GRB 091024 (Gruber et al. 2011, with $T_{90} \approx 1020 \mathrm{~s}$ ) which is also associated with an optical flash (Virgili et al. 2013), the dark GRB 090417B (Holland et al. 2010, $T_{90}>2130$ s), the "double burst" GRB 110709B (Zhang et al. 2012, with a total duration of $\approx 1400 \mathrm{~s}$ ) and the similar GRB 121217A (Siegel et al. 2013; Elliott et al. 2014, with a total duration of $>1070$ s), the "Swift Birthday Burst" GRB 141121A (Golenetskii et al. 2014; Cucchiara et al. 2015 , with a total duration of $\approx 1410 \mathrm{~s}$ ), and, somewhat shorter, GRB 070616 (Starling et al. 2008), which lasted $\approx 600 \mathrm{~s}$.

Since GRB 111209A, three very similar events have been discovered. GRB 121027A features a highly variable X-ray light curve and extremely elevated emission at $>5000 \mathrm{~s}$ after the trigger (Serino et al. 2012; Evans et al. 2012, see Peng et al. 2013; Wu et al. 2013 and Hou et al. 2014 for theoretical treatments), which, combined with the redshift $z=1.773$, makes it one of the most luminous X-ray afterglows ever detected (L14, Starling et al., in prep.). L14 list this as a third example of an ultra-long duration GRB (see below for the other event).

Not included in the sample of L14, GRB 130925A (Evans et al. 2014; Zhang et al. 2014) was found to show flaring emission, both in gamma-rays (Fitzpatrick 2013; Markwardt et al. 2013; Savchenko et al. 2013;

15 http://wWw . ioffe.rssi .ru/LEA/GRBs/GRB111209A/ 
Golenetskii et al. 2013; Hurley et al. 2013; Evans et al. 2014) as well as X-rays (Suzuki et al. 2013; Evans et al. 2013), for a duration of over $12000 \mathrm{~s}$, starting with a short, soft precursor, followed by two large emission episodes and several further soft flares. While an initial similarity to $\mathrm{Sw} \mathrm{J} 1644+57$ was remarked upon (Burrows et al. 2013), not only due to the high-energy emission, but also due to the highly obscured optical counterpart (Sudilovsky et al. 2013a; Greiner et al. 2014) and the very similar redshift (Vreeswijk et al. 2013; Sudilovsky et al. 2013b), the later evolution of the X-ray afterglow as well as the offset from the host galaxy centre (Tanvir et al. 2013) point to this being another ULGRB and not a (relativistic) TDE. Similar to GRB 111209A, the early optical/NIR emission during the prompt phase shows strong variability as well as a multi-100 s offset between gamma-rays and optical light (Greiner et al. 2014). The prompt emission itself is much more variable than that of GRB 111209A, though, and does not look "stretched" (Evans et al. 2014; Greiner et al. 2014). The late X-ray afterglow, for which spectral features have been claimed (Bellm et al. 2014, but see Evans et al. 2014), is extremely soft, which has been explained as an expanding dust scattering halo (Evans et al. 2014; Zhao \& Shao 2014; Margutti et al. 2015), or alternatively as thermal emission from a hot cocoon, which may be a link to BSG progenitors (Piro et al. 2014; Basak \& Rao 2015, but see Evans et al. 2014). In strong contrast to the host of GRB 111209A, the host of GRB 130925A shows super-solar metallicities at multiple sites, including the explosion site (Schady et al. 2015). This event also features a peculiar radio afterglow with unique properties (Horesh et al. 2015). We discuss the optical/NIR afterglow light curve in more detail in Sect. 3.4 and compare it to that of GRB 111209A in Sect. 4.3. The newest member of the ULGRB class is also the longest so far, the "Bastille Day" GRB 170714B. It was localized by Swift (D'Ai et al. 2017), and in this case, the BAT data indicates that it was caught almost at onset (Palmer et al. 2017), with detectable emission beginning just $70 \mathrm{~s}$ before the beginning of the image trigger. Initial X-ray observations were reported to show a lack of variability (D'Avanzo et al. 2017) but after further observations, Kann et al. (2017) pointed out that the X-ray light curve showed clear signs of an ULGRB, with a duration of at least $36 \mathrm{ks}$. GTC observations revealed a faint, very reddened afterglow (de Ugarte Postigo et al. 2017a), and GTC spectroscopy revealed the GRB to lie at $z=0.793$ (de Ugarte Postigo et al. 2017b). Radio follow-up was initially unsuccessful (de Ugarte Postigo et al. 2017c; Ricci et al. 2017; Horesh et al. 2017), but a faint afterglow was finally detected in further observations (Piro et al. 2017). Such a faint radio afterglow is a strong indicator that this is not a relativistic TDE (de Ugarte Postigo et al. 2017c). No detection of an accompanying SN has been reported so far. Hou et al. (2018) model the early X-ray emission with a quark-star model.

- "Precursor GRBs" exhibit a comparatively faint first emission episode (which is spectrally similar to the main emission Burlon et al. 2008) followed by a "silence" that can last hundreds of seconds before a much more luminous emission episode occurs which contains most of the fluence of the GRB and can last for several hundred seconds further. A "short" example of such a GRB is GRB 061121
(Page et al. 2007), and the four well-known long cases are GRB 041219A (main emission after $\approx 200 \mathrm{~s}$, total duration $\approx 500 \mathrm{~s}$, Fenimore et al. 2004; Blake et al. 2005), GRB 050820A (main emission after $\approx 220 \mathrm{~s}$, total duration $\approx 600$ s, Cenko et al. 2006), GRB 060124 (main emission after $\approx 500 \mathrm{~s}$, total duration $\approx 750 \mathrm{~s}$, Romano et al. 2006), and GRB $160625 \mathrm{~B}$ (main emission after $\approx 180 \mathrm{~s}$, total duration $\approx 800$ s, Zhang et al. 2018); in the latter case, the long duration and delayed main emission allowed strong polarization to be detected during the prompt emission (Troja et al. 2017).

- A seemingly very rare class of EL-GRBs are single-peak (Fast Rise, Exponential Decline, FRED) hard events. Only two have been reported so far: GRB 971208 detected by BATSE and Konus-WIND (Connaughton et al. 1997, duration $\approx 2500 \mathrm{~s}$ ), and the extreme event GRB 060814B, which lasted for over four hours in the softest Konus-WIND bands (Pal'Shin et al. 2008).

- Morphologically similar but spectrally distinct are lowluminosity, low-redshift X-Ray Flashes (XRFs), which are associated with broad-lined Type Ic supernovae. Two of these have been extensively studied: XRF 060218 (Campana et al. 2006, duration $\approx 2100 \mathrm{~s}$ ) associated with SN 2006aj (Pian et al. 2006; Mirabal et al. 2006; Modjaz et al. 2006; Cobb et al. 2006; Sollerman et al. 2006; Ferrero et al. 2006), and XRF 100316D (Starling et al. 2011, duration $>1300$ s), associated with SN 2010bh (Chornock et al. 2010; Cano et al. 2011; Olivares E. et al. 2012; Bufano et al. 2012; Margutti et al. 2013). The long duration of these events is likely due to additional emission components such as thermal emission from a supernova shock breakout (e.g. Campana et al. 2006; Starling et al. 2012).

- Finally, there are high-energy transients which resemble GRBs but are possibly due to a different type of progenitor. The "Christmas Burst" GRB 101225A (with a duration of several thousand seconds, and possibly even days) has been explained as the inspiral of a neutron star into a helium star, creating a central engine similar to a GRB (Thöne et al. 2011) ${ }^{16}$. L14 list this as the first ULGRB discovered, though it shows behaviour especially in the optical which is unlike any known GRB (Thöne et al. 2011), in contrast to GRBs 111209A, 121027A, and 130925A. Finally, the extreme gamma-ray transients GRB 110328A/Swift J164449.3+573451 (Levan et al. 2011; Bloom et al. 2011; Burrows et al. 2011; Zauderer et al. 2011), Swift J2058.4+0516 (Cenko et al. 2012; Pasham et al. 2015), and Swift J1112.2-8238 (Brown et al. 2015, 2017) which have been detected for many days at high energies, are very likely due to the tidal disruption of a star by a supermassive black hole, which "turns on" a blazar-like relativistic jet.

We note that Virgili et al. (2013) undertake a more broad classification, discerning between "Interrupted Emission" and "Continuous Emission" GRBs. The Interrupted Emission GRBs are all found in our first category. These authors also give details on many of the EL-GRBs we list above in the appendix of their work.

${ }^{16}$ We note that a non-cosmological origin has been proposed as well (Campana et al. 2011), but this has now been firmly ruled out by the derivation of the host-galaxy redshift $z=0.847$ (L14), which is incidentally also significantly higher than the distance used in Thöne et al. (2011), $z \approx 0.33$. 